\title{
Non-oscillatory forward-in-time integrators for viscous incompressible flows past a sphere
}

\author{
Joanna Szmelter ${ }^{\mathrm{a}}$, Piotr K. Smolarkiewicz ${ }^{\mathrm{b}}$, Zhao Zhang ${ }^{\mathrm{c}}$, \\ Zhixin $\mathrm{CaO}^{\text {a }}$ \\ ${ }^{a}$ Loughborough University, Leicestershire LE11 3TU, UK \\ ${ }^{\mathrm{b}}$ European Centre For Medium Range Weather Forecasts, Reading, RG2 9AX, UK \\ ${ }^{\mathrm{c}}$ Heriot-Watt University, Edinburgh, EH14 4AS, UK
}

\begin{abstract}
A non-oscillatory forward-in-time (NFT) integrator is developed to provide solutions of the Navier-Stokes equations for incompressible flows. Simulations of flows past a sphere are chosen as a benchmark representative of a class of engineering flows past obstacles. The methodology is further extended to moderate Reynolds number, stably stratified flows under gravity, for Froude numbers that typify the characteristic regimes of natural flows past distinct isolated features of topography in weather and climate models. The key elements of the proposed method consist of the Multidimensional Positive Definite Advection Transport Algorithm (MPDATA) and a robust non-symmetric Krylov-subspace elliptic solver. The solutions employ a finite volume spatial discretisation on unstructured and hybrid meshes and benefit from a collocated arrangement of all flow variables while being inherently stable. The development includes the implementation of viscous terms with the detachededdy simulation (DES) approach employed for turbulent flows. Results demonstrate that the proposed methodology enables direct comparisons of the numerical solutions with corresponding laboratory studies of viscous and stratified flows while illustrating accuracy, robustness and flexibility of the NFT schemes. The presented simulations also offer a better insight into stably stratified flows past a sphere.
\end{abstract}

Key words: Multidimensional high resolution schemes, incompressible viscous flows, stably stratified flows, MPDATA, anelastic flows, upwind approximations PACS:

* Corresponding Author.
Email address: j.szmelter@lboro.ac.uk (Joanna Szmelter).

Preprint submitted to J. Comput. Phys.

11 February 2019 


\section{INTRODUCTION}

External incompressible flows past immersed bodies have numerous applications in fluid dynamics and engineering. Despite its geometric simplicity, the sphere plays an important role in a study of such flows. Incompressible flows past the sphere can involve an abundance of complex phenomena that are of general importance, and the research into numerical methods resolving them adequately at a range of regimes is on-going. This interest extends to density stratified flows, since the experimental studies of flows past a sphere are traditionally used to gain insights into physics common to atmospheric flows past orography, also relevant to oceanographic applications.

Finite-volume discretisations on hybrid meshes combining prismatic elements near solid boundaries and tetrahedra elements elsewhere have become increasingly popular in simulations of viscous engineering flows [22]. In contrast, although unstructured meshes have been a subject of meteorological interest for some time - see [58] for an extended overview - their intrinsic flexibility, have been put to practical use only at the beginning of the present century, in the context of forecasting high-impact weather, air quality and environmental hazard [2]. Since then, there has been a continuously growing research into modelling atmospheric flows on unstructured meshes. In comparison to the established finite-difference techniques, the finite volume discretisations are not constrained to rigid connectivity of regular grids. This flexibility turns particularly instrumental for the advancement of global atmospheric models for weather and climate with quasi uniform horizontal resolution supporting both the finite volumes and spherical harmonics [57]. However, benefits of the flexible meshing are not limited to global flows, and they can be equally important at finer-scale problems of computational meteorology; for example, in research and forecasting of weather in mountainous areas or over island archipelagoes. In their very essence, such problems can be thought of as flows past blunt bodies, among which a flow past a sphere historically plays a special rolecomplementary to a flow past a cylinder - and comprises a large portfolio of works employing theoretical, experimental and numerical methods $[1,5,16,13]$.

Many schemes, especially those building on the pressure correction approach, have been devised as solution methods for the Navier-Stokes equations governing incompressible viscous flows. However, the application of the nonoscillatory forward-in-time, NFT, integrators is new in this context. The NFT integrators were proposed in the early nineteen nineties for problems related to geophysical flows [49], as a class of fully second-order-accurate, either semiLagrangian or Eulerian, algorithms built on two-time-level nonlinear advection techniques that control numerical oscillations characteristic of higher-order linear schemes. The NFT solvers were originally developed for finite-difference discretisations on structured grids and only recently their applicability was 
extended to finite-volume discretisation on unstructured meshes, giving rise to a class of NFTFV integrators. The initial developments of unstructured meshes based NFTFV schemes resulted in inviscid compressible flow models, documented for supersonic, transonic and subsonic aerodynamics [52,53]. Further developments focused on atmospheric flows, and included a selection of nonhydrostatic models for mesoscale orographic flows, with a range of internal gravity wave phenomena in two and three spatial dimensions $[65,54,56]$, generalisations of shallow-water equations to global spherical geometry [64] and a class of three dimensional global hydrostatic models $[64,65]$.

All of these NFTFV schemes use the unstructured meshes based MPDATA $[50,51,24]$ for the nonlinear advection operator. This choice provides several benefits. Apart from the MPDATA's sign-preservation property - one of the primary requirements of atmospheric modelling - the algorithm also provides inherent ability to control numerical oscillations resulting, e.g., from discontinuous representation of shock waves in compressible flows [53] or use of non-staggered meshes for incompressible flows. In the implementations presented here all dependent flow variables are collocated, to lower memory and communication requirements compared to staggered arrangements as well as to facilitate the design of elaborate semi-implicit time integrations schemes $[57,58,24,59]$. For incompressible engineering flows and anelastic atmospheric flows, instabilities arise with the collocated variables from the coupling between velocity and pressure. In the NFT schemes these are regularised by MPDATA working in synergy with an implicit solution of the Poisson equation for the pressure perturbation. Subsequently, the schemes allow a construction of low-numerical-diffusion models which are nonlinearly stable. Another useful feature of these schemes is the direct availability of the leading truncation error compensated by MPDATA, which can be exploited for the purpose of constructing error indicators for mesh adaptivity $[63,66]$.

A further benefit that MPDATA brings to the NFT schemes is its proven implicit large eddy simulation (ILES) capability [31,10,37,55]. This by-product of the MPDATA iterative apparatus reverses the filtering operation of the low-order upwinding, leaving the leading-order truncation terms dissipative and acting as a higher-order filter at the limit of grid resolution. This selfregularisation property allows for a quality large-eddy simulation without invoking subgrid scale models, thus eliminating the need of evaluating viscous stress for high Reynolds number flows [37]. Examples of ILES with the NFTFV integrators applied to atmospheric flows are included in [66]. An important verification of such ILES was provided in [56], by comparing NFTFV results with the results from explicit LES on structured grids, in simulations of convective planetary boundary layer epitomising high-Reynolds-number stochastic flows with predicable integral characteristics.

In this paper, we complement our earlier work with new developments of 
NFTFV models that provide capabilities for simulation of viscous effects for solutions obtained on unstructured/hybrid meshes. While the successful performance of ILES has been documented for stratified geophysical flows, this work explores alternative methodologies for providing means of including viscous effects and explicit turbulence modelling required for simulating incompressible and anelastic flows through a range of Reynolds number regimes. The implementation of the NFTFV integrator is presented in the context of a general scheme that is suitable for simulating the incompressible Navier-Stokes equations with constant mass density, and the anelastic Lipps-Hemler equations $[27,28]$ with significant vertical variations of a static environment. The resulting NFTFV integrator exploits the mathematical commonalities of the two PDE systems, namely the diagnostic form of the mass continuity equation and the operator form of the viscous forcings. The focus of the Navier-Stokes solver is on the solution dependence on the Reynolds number, $\operatorname{Re}\left(=V_{o} L / \nu\right)$ where $V_{o}$ and $\nu$ refer to free stream velocity and kinematic viscosity, while $L$ is the characteristic length scale of the problem. The focus of the anelastic solver is on the solution dependence on the Froude number, $\operatorname{Fr}\left(=V_{o} / N h\right)$ with $N$ and $h$ to be defined shortly. The two numbers characterise the relative impact of the dominant right-hand-side (rhs) forcing on the mass flow, by providing the ratio of the characteristic time scales of the viscous and buoyant forcings to the time scale of inertia, respectively, for the Navier-Stokes and Lipps-Hemler equations. Having a common solver for both systems, particularly benefits the atmospheric applications.

Natural flows past topography are subject to intricate realisations largely depending on the Froude number. Even for the elementary uniform profiles of ambient buoyancy frequency $N$ and velocity $V_{o}$, such canonical flows vary from 3D potential flow over the obstacle with height $h$, as $\operatorname{Fr} \nearrow \infty$, to quasi 2D potential flow around it, as $F r \searrow 0$. In between, more complex responses comprise gravity waves aloft, obstacle-scale eddies in the lee and the upwind blocking. Apart from verifying new capabilities of NFTFV MPDATA class of solvers for engineering applications, the aim of the present development is to provide a reliable tool capable of shedding light on the long-lasting controversy on the relative roles of viscosity and density stratification in generating the intricate solutions throughout the high to low $F r$ regimes. The objective of the development is to harness the flexibility of the NFTFV integrators to enable direct comparisons of the numerical solutions with corresponding laboratory studies of viscous, stably stratified flows past a sphere. The presented numerical results offer new insights, while demonstrating the accuracy, robustness and flexibility of the NFT schemes.

The remainder of the paper is organized as follows. In the next section, the outline of the NFTFV integrator for viscous flows and its implementation on unstructured and hybrid meshes is provided. A systematic numerical study of flows past a sphere at a range of Reynolds numbers is documented in Section 4. 
Section 5 reports on an investigation of a sphere moving horizontally in a stratified laminar flow. Remarks in Section 6 conclude the paper.

\section{GOVERNING EQUATIONS}

\subsection{Incompressible Navier-Stokes equations}

The incompressible Navier-Stokes equations for isothermal flows can be compactly written as

$$
\begin{aligned}
& \nabla \cdot\left(\mathbf{V} \rho_{o}\right)=0, \\
& \frac{\partial \rho_{o} V_{I}}{\partial t}+\nabla \cdot\left(\mathbf{V} \rho_{o} V_{I}\right)=-\rho_{o} \frac{\partial \varphi}{\partial x_{I}}+(\nabla \cdot \boldsymbol{\tau})_{I},
\end{aligned}
$$

where $V_{I(=1,2,3)}$ denote the velocity components in $x_{I}$ Cartesian coordinate directions, $\rho_{o}$ is a constant reference density, and $\varphi=\left(p-p_{e}\right) / \rho_{o}$ is the densitynormalized pressure perturbation with respect to static environmental profile $p_{e}$. Herein we consider small laboratory scales such that the reference density can be assumed constant. The entries of the deviatoric stress tensor $\boldsymbol{\tau}$ in (1) are given by

$$
\tau_{I J}=\left(\mu+\mu_{T}\right)\left(\frac{\partial V_{I}}{\partial x_{J}}+\frac{\partial V_{J}}{\partial x_{I}}\right)
$$

where $\mu$ is the dynamic viscosity of the fluid, and the dynamic eddy viscosity $\mu_{T}$ has a flexible meaning, switching between a Reynolds-Averaged NavierStokes (RANS) turbulence model with $\mu_{T}=\mu_{t}$ or an LES turbulence model with a subgrid-scale viscosity $\mu_{T}=\mu_{\text {sgs }}$.

For simulation of turbulent incompressible flows we adopt detached-eddy simulation (DES) approach originally proposed by Spalart [60]. DES is a hybrid of RANS and LES, where RANS applies to most of the boundary layer region near the solid walls, while LES is employed in the remaining part of the computational domain. Consequently in the governing equations the flow variables are considered to be either time-averaged in RANS computational zones or spatially filtered in LES zones. In our implementation, the dynamic eddy viscosity $\mu_{t}$ is approximated using the modified Spalart-Allmaras model [61]. In particular,

$$
\mu_{t}=\rho_{o} \hat{\nu} f_{v 1}, \quad \text { with } f_{v 1}=\frac{\chi^{3}}{\chi^{3}+c_{v 1}^{3}}, \quad \chi=\frac{\hat{\nu}}{\nu}, \text { and } \nu=\frac{\mu}{\rho_{o}}
$$


where $\nu$ is the kinematic viscosity, and $\hat{\nu}$ is prognosed by the transport equation

$$
\frac{\partial \hat{\nu}}{\partial t}+\mathbf{V} \cdot \nabla \hat{\nu}=c_{b 1} \hat{S} \hat{\nu}+\frac{1}{\sigma} \nabla \cdot[(\nu+\hat{\nu}) \nabla \hat{\nu}]+\frac{c_{b 2}}{\sigma}(\nabla \hat{\nu})^{2}-c_{w 1} f_{w}\left(\frac{\hat{\nu}}{d}\right)^{2}
$$

Here

$$
\hat{S}=S+\frac{\hat{\nu}}{\kappa^{2} d^{2}} f_{v 2}, \quad f_{v 2}=1-\frac{\chi}{1+\chi f_{v 1}}, \quad S=\left(2 \sum_{I, J=1,3} \Omega_{I J} \Omega_{I J}\right)^{1 / 2},
$$

where the entries of the rotation tensor are given by

$$
\Omega_{I J}=\frac{1}{2}\left(\frac{\partial V_{I}}{\partial x_{J}}-\frac{\partial V_{J}}{\partial x_{I}}\right)
$$

and the remaining coefficient are specified as

$$
f_{w}=\gamma\left(\frac{1+c_{w 3}^{6}}{\gamma^{6}+c_{w 3}^{6}}\right)^{1 / 6}, \gamma=r+c_{w 2}\left(r^{6}-r\right), \quad r=\frac{\hat{\nu}}{\hat{S} \kappa^{2} d^{2}}
$$

Furthermore, the constants appearing in (3)-(7) are set as

$$
\begin{aligned}
& c_{b 1}=0.1355, \quad \sigma=2 / 3, \quad c_{b 2}=0.622, \quad \kappa=0.41, \\
& c_{w 1}=c_{b 1} / \kappa^{2}+\left(1+c_{b 2}\right) / \sigma, \quad c_{w 2}=0.3, \quad c_{w 3}=2.0, \quad c_{v 1}=7.1 .
\end{aligned}
$$

The parameter $d$ appearing in (4), (5) and (7) has dual meaning. In RANS $d=d_{w}$, where $d_{w}$ is the actual distance to the nearest wall, whereas in DES $d=\min \left(d_{w}, C_{D E S} \Delta\right)$, where $C_{D E S}=0.65$ is used after [42] and [5]. In the adopted finite volume method, the dual mesh cell size naturally limits the cut-off width in the spatial filter; therefore, in our calculations $\Delta$ is estimated according to $\Delta=\sqrt[3]{\mathcal{V}_{P}}$, where $\mathcal{V}_{P}$ is the dual cell volume encompassing mesh node $P$.

\subsection{Anelastic Lipps-Hemler equations}

Next we consider the Lipps-Hemler $[27,28]$ anelastic system (9). The governing conservation laws of mass, momentum and entropy fluctuations ${ }^{1}$ are taken in the Boussinesq limit - mathematically isomorphic for stratified flows

\footnotetext{
${ }^{1}$ For the ideal gas, the specific entropy $s$ and the potential temperature $\theta \equiv$ $T\left(p / p_{o}\right)^{-R / c_{p}}$ are related via $d s=c_{p} d \ln \theta$, with the usual symbols $T, p, p_{o}, R$ and $c_{p}$ corresponding to temperature, pressure, constant reference pressure, gas constant and specific heat at constant pressure.
} 
of ideal gas and saline solution - unifying the atmospheric, oceanic and laboratory interest in the flows past a sphere investigated numerically in Section 5. Reminiscent of the incompressible system (1), these anelastic equations are compactly expressed as

$$
\begin{aligned}
& \nabla \cdot\left(\mathbf{V} \rho_{o}\right)=0 \\
& \frac{\partial \rho_{o} V_{I}}{\partial t}+\nabla \cdot\left(\mathbf{V} \rho_{o} V_{I}\right)=-\rho_{o} \frac{\partial \varphi}{\partial x_{I}}+\rho_{o} g \frac{\theta^{\prime}}{\theta_{o}} \delta_{I 3}+(\nabla \cdot \boldsymbol{\tau})_{I}, \\
& \frac{\partial \rho_{o} \theta^{\prime}}{\partial t}+\nabla \cdot\left(\mathbf{V} \rho_{o} \theta^{\prime}\right)=-\mathbf{V} \cdot \nabla \theta_{\mathbf{e}} .
\end{aligned}
$$

Here, the perturbation pressure is meant as $\varphi=c_{p} \theta_{o}\left(\pi-\pi_{e}\right)$, where $\pi \equiv$ $\left(p / p_{o}\right)^{R / c_{p}}$ denotes the Exner-function of pressure. For the ideal gas, the formulae $\rho^{-1} \nabla p=c_{p} \theta \nabla \pi$ and $\pi=T / \theta$ interrelate (1) and (9). Unlike (1), the anelastic system accounts for the gravitational acceleration $g$ via the buoyancy term on the rhs of the momentum equation (9b), in which $\theta^{\prime}=\theta-\theta_{e}$ is the potential temperature perturbation about a static ambient profile $\theta_{e}\left(x_{3}\right)=\theta_{o}+S_{o} x_{3}$, with $S_{o}$ marking a constant stratification, and $\delta_{I J}$ denotes the Kronecker delta. The reference constants $\rho_{o}, p_{o}$ and $\theta_{o}$ are assumed to coincide with surface values of the ambient profiles. They enter the mass continuity equation (9a), the definition of the pressure perturbation as well as the denominator of the buoyancy term in the momentum equation - in effect of the assumed Boussinesq limit of the anelastic approximation. In a more general Lipps-Hemler system, these reference values would be replaced with the vertically varying reference profiles. The entropy equation $(9 \mathrm{c})$ is equivalent to assuming adiabatic motions with potential temperature constant along a flow trajectory, $d \theta / d t=0$. The diffusion of heat is neglected, consistently with the laboratory experiments and their related numerical simulations $[29,15]$ conducted in saline solutions with large Schmidt numbers. The perturbation forms of the momentum and thermodynamic equation, cf. [54], are chosen to simplify the implementation of initial and boundary conditions and to benefit the solution accuracy.

\section{NUMERICAL METHOD}

\subsection{A semi-implicit, non-oscillatory forward-in-time finite-volume (NFTFV) integrator}

In the proposed development, the numerical integrator is designed for the general anelastic system of PDEs-EQS. 9 with $\rho_{o}, \pi_{o}$ and $\theta_{o}$ being reference profiles in function of $x_{3}$ rather than reference constants - with the Boussinesq and incompressible PDEs realised as options. 
The spatial discretisation follows the finite volume implementation using median dual meshes with edge-based connectivity, detailed in $[51,66]$, proven suitable for integrating the governing PDEs over arbitrary shaped irregular polyhedral cells. Figure 1 shows a schematic of the edge connecting mesh node $P$ with one of its immediate neighbours $Q$, and the corresponding face $S_{q}$ of the dual cell surrounding point $P$. A dual cell is constructed by joining centres of the edges with the barycentres of polyhedra and faces of the primary mesh elements sharing edge $P-Q$. All dependent variables are collocated in the nodes exemplified by $\mathrm{P}$ and $\mathrm{Q}$.

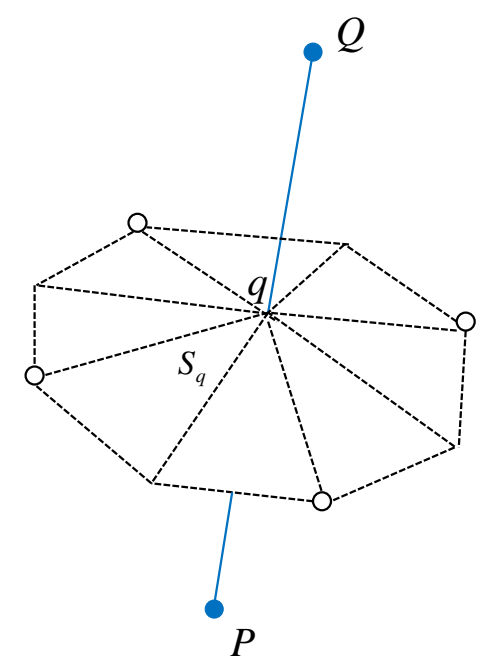

Fig. 1. The median-dual cell face in 3D. The edge connecting nodes $P$ and $Q$ pierces (at the edge centre $q$ ) the face $S_{q}$ of a computational (dual) cell surrounding node $\mathrm{P}$; open circles represent barycenters of polyhedral elements from the primary mesh sharing the edge. Dashed lines mark a cell face of the dual mesh.

Each of the PDEs in the system (9) can be written in a form of a generalised inhomogeneous transport equation for a specific scalar variable $\xi$ advected with the vector field $\boldsymbol{V}$ and the rhs subsuming all inviscid and dissipative forcing/source terms,

$$
\frac{\partial \rho_{o} \xi}{\partial t}+\nabla \cdot\left(\mathcal{V} \rho_{o} \xi\right)=\rho_{o} R
$$

where $\rho_{o} R$ amounts to the right-hand-sides of the prognostic PDEs in (9). After [49], (10) can be integrated to the second-order accuracy in time and space with the non-oscillatory forward-in-time (NFT) algorithm

$$
\begin{aligned}
\xi_{P}^{n+1} & =\mathcal{A}_{P}\left(\xi^{n}+0.5 \delta t R^{n}, \mathcal{V}^{n+1 / 2}, \rho_{o}\right)+0.5 \delta t R_{P}^{n+1} \\
& \equiv \mathcal{A}_{P}\left(\widetilde{\xi}, \mathcal{V}^{n+1 / 2}, \rho_{o}\right)+0.5 \delta t R_{P}^{n+1} \equiv \widehat{\xi}_{P}+0.5 \delta t R_{P}^{n+1}
\end{aligned}
$$


where $n$ and $P$ refer, respectively, to an arbitrary instant and node location on the mesh. The operator $\mathcal{A}$ is a shorthand for the edge-based option of MPDATA [51], and it already accounts for a division of the discrete integral of (10) by $\rho_{o}$. Furthermore, $R^{n+1}$ is a second-order-accurate finite volume representation of $R$; whereas the $\mathcal{V}^{n+1 / 2}$ argument of $\mathcal{A}$ denotes an $\mathcal{O}\left(\delta t^{2}\right)$ estimate of $\rho_{o} \mathbf{V}$ at $t+0.5 \delta t$. Herein, $\mathbf{V}^{n+1 / 2}$ is linearly extrapolated from $\mathbf{V}^{n-1}$ and $\mathbf{V}^{n}$, thus assuring vanishing of the discrete $\nabla \cdot\left(\mathbf{V} \rho_{o}\right)$, given it vanished at the $n-1$ and $n$ instants. Notably, the algorithm in (11) is congruent with the semi-Lagrangian, trajectory-wise trapezoidal integral of the ODE $d \xi / d t=R$ that underlies the Eulerian conservation form (10).

The derivation of our NFTFV integrator for the entire system (9) starts with extending the NFT template (11) to the prognostic equations for all velocity components $V_{I}$ and $\theta^{\prime}$ :

$$
\begin{aligned}
V_{1}^{n+1} & =\widehat{V}_{1}-0.5 \delta t{\frac{\partial \varphi}{\partial x_{1}}}^{n+1} \\
V_{2}^{n+1} & =\widehat{V}_{2}-0.5 \delta t{\frac{\partial \varphi}{\partial x_{2}}}^{n+1} \\
V_{3}^{n+1} & =\widehat{V}_{3}-0.5 \delta t{\frac{\partial \varphi}{\partial x_{3}}}^{n+1}-0.5 \delta t g{\frac{\theta^{\prime}}{\theta_{o}}}^{n+1} \\
\theta^{\prime n+1} & =\widehat{\theta}^{\prime}-0.5 \delta t \frac{\partial \theta_{e}}{\partial x_{3}} V_{3}^{n+1}
\end{aligned}
$$

where $V_{I}^{n+1}$ and $\theta^{\prime n+1}$ are the unknown solutions at the instant $t^{n+1}$ and node $P$. Because all the terms in (12) are evaluated consistently at the same $P$ location, the symbol $P$ is dropped for conciseness. It will be omitted in the remainder of the paper, unless desired for clarity. Furthermore, the auxiliary fields $\widetilde{V_{I}}$ and $\widetilde{\theta^{\prime}}$ of the respective explicit counterparts $\widehat{V}_{I}$ and $\widehat{\theta}^{\prime}$ of the solution in (12) are written as

$$
\begin{aligned}
& \widetilde{V_{1}}=V_{1}^{n}+0.5 \delta t\left(-\frac{\partial \varphi}{\partial x_{1}}+\frac{2}{\rho_{o}} \sum_{J=1,3} \frac{\partial \tau_{1 J}}{\partial x_{J}}\right)^{n}, \\
& \widetilde{V_{2}}=V_{2}^{n}+0.5 \delta t\left(-\frac{\partial \varphi}{\partial x_{2}}+\frac{2}{\rho_{o}} \sum_{J=1,3} \frac{\partial \tau_{2 J}}{\partial x_{J}}\right)^{n}, \\
& \widetilde{V_{3}}=V_{3}^{n}+0.5 \delta t\left(-\frac{\partial \varphi}{\partial x_{3}}+g \frac{\theta^{\prime}}{\theta_{o}}+\frac{2}{\rho_{o}} \sum_{J=1,3} \frac{\partial \tau_{3 J}}{\partial x_{J}}\right)^{n}, \\
& \widetilde{\theta}^{\prime}=\theta^{\prime n}-0.5 \delta t \frac{\partial \theta_{e}}{\partial x_{3}} V_{3}^{n} .
\end{aligned}
$$

The system (13) modifies the template (11), in that the viscous forcing terms are integrated explicitly to $\mathcal{O}\left(\delta t^{2}\right)$, in the manner congruent with the Eulerforward semi-Lagrangian integral along the flow trajectory $[53,55]$. The latter is manifested by the factor 2 in front of the stress divergence. 


\subsection{A discrete Poisson boundary-value problem (BVP)}

The system (12), supplied with a discretised mass continuity equation (9a) taken at $t^{n+1}$ and all nodes $P$

$$
\forall P \quad\left(\sum_{J=1,3} \frac{\partial \rho_{o} V_{J}^{n+1}}{\partial x_{J}}\right)_{P}=0,
$$

is implicit because all five dependent variables are unknown at $t^{n+1}$ and depend on each other. However due to the collocated data arrangement, further solution procedure is fairly straightforward. Starting with substitution of (12d) into the buoyancy term in (12c), modifies the latter into

$$
V_{3}^{n+1}=\left(\widehat{V_{3}}+0.5 \delta t \frac{g}{\theta_{o}}{\widehat{\theta^{\prime}}}^{-0.5 \delta t} \frac{\partial \varphi^{n+1}}{\partial x_{3}}\right) /\left(1+(0.5 \delta t)^{2} \frac{g}{\theta_{o}} \frac{\partial \theta_{e}}{\partial x_{3}}\right) .
$$

The inspection of (15) inspires streamlining the presentation, by defining the auxiliary coefficients (or more generally fields thereof)

$$
\alpha_{I}=\frac{g}{\theta_{o}} \delta_{I 3}, \text { and } \beta_{I}=1+(0.5 \delta t)^{2} \alpha_{I} \frac{\partial \theta_{e}}{\partial x_{3}}
$$

that apart from $\alpha_{3}$ and $\beta_{3}$, take values $\alpha_{1}=\alpha_{2}=0$ and $\beta_{1}=\beta_{2}=1$. Given (16), the velocity updates (12a)-(12c) can be rewritten as

$$
V_{I}^{n+1}=\widehat{\widehat{V}_{I}}-0.5 \delta t \beta_{I}^{-1}{\frac{\partial \varphi}{\partial x_{I}}}^{n+1}
$$

where

$$
\widehat{\widehat{V_{I}}}=\beta_{I}^{-1}\left(\widehat{V_{I}}+0.5 \delta t \alpha_{I} \widehat{\theta^{\prime}}\right)
$$

For $J=1,2,(18)$ implies $\widehat{\widehat{V}_{J}} \equiv \widehat{V_{J}}$, upon which (17) reproduces (12a) and (12b), respectively. However, for $J=3$, (17) amounts to (15). Consequently, substituting (17) into the discretised mass continuity equation (14) leads to a discrete elliptic Poisson equation for $\varphi^{n+1}$

$$
\forall P \quad\left\{\sum_{J=1,3} \frac{\partial}{\partial x_{J}}\left[\rho_{o}\left(\widehat{\widehat{V}}_{J}-0.5 \delta t \beta_{J}^{-1}{\frac{\partial \varphi}{\partial x_{J}}}^{n+1}\right)\right]\right\}_{P}=0 .
$$

The BVP (19) is solved using the generalised conjugate residual approach detailed in [54], subject to the Neumann boundary conditions for $\varphi^{n+1}$ implied by the Dirichlet boundary conditions for $\mathbf{V}^{n+1}$

$$
\mathbf{V}^{n+1} \cdot \mathbf{n}=\left(\widehat{\widehat{\mathbf{V}}}-0.5 \delta t \boldsymbol{\beta}^{-1} \nabla \varphi^{n+1}\right) \cdot \mathbf{n}=\mathbf{V}_{B}^{\perp}
$$


Here $\boldsymbol{\beta}^{-1}$ is the $3 \times 3$ diagonal matrix with the entries equal to reciprocals of $\beta_{I}$. Furthermore, $\mathbf{V}_{B}^{\perp}$ refers to a normal flow specified at the boundary $\partial \boldsymbol{\Omega}$ of the integration domain $\boldsymbol{\Omega}$, and $\mathbf{n}$ marks the outward unit normal to $\partial \boldsymbol{\Omega}$. By construction $\mathbf{V}_{B}^{\perp}$ satisfies the integrability condition $\oint_{\partial \boldsymbol{\Omega}} \rho_{o} \mathbf{V}_{B}^{\perp} d \sigma=0$ to machine precision, and so (20) implies this same for $\mathbf{V}^{n+1}$. The results discussed later in the paper satisfy (19) with tolerance $\epsilon$ defined in terms of the BVP's residual errors as

$$
\|\delta t \times \operatorname{lhs}(\mathrm{EQ} .19)\|_{\infty}<\epsilon \mathcal{C}
$$

with a typical $\epsilon=10^{-5}$ assuming maximal Courant numbers $\mathcal{C} \lesssim 1$; see $\S 4.3$ in [55] for an extended discussion.

\subsection{Implementation}

\subsubsection{Advection operator}

Evaluating the action of the advective-transport operator $\mathcal{A}$ on the auxiliary variable $\widetilde{\xi}$ in $(11)$ is a most elaborate part of the model code, comparable in complexity to the elliptic solver. Various versions of MPDATA were documented in the literature over the last three decades. Here we give the key details of the "infinite-gauge" option used solely in this study. The crux of the gauge option is a linearisation of the basic MPDATA about an infinite constant background. The presented scheme derives from the unstructured-mesh MPDATA proposed in [51].

Given the arguments $\widetilde{\xi}, \boldsymbol{V}^{n+1 / 2}$ and $\rho_{o}$, the procedure that forms the $\mathcal{A}_{P}(., .,$. term in (11) commences with calculating the upwind fluxes normal to the cell face $S_{q}$ (see Fig. 1 for the cell geometry and symbols)

$$
F_{q}^{\perp}=\left[v_{q}^{\perp}\right]^{+} \widetilde{\xi}_{P}+\left[v_{q}^{\perp}\right]^{-} \widetilde{\xi}_{Q}
$$

where

$$
v_{q}^{\perp}=\mathbf{S}_{q} \cdot 0.5\left[\boldsymbol{\nu}_{Q}^{n+1 / 2}+\mathcal{V}_{P}^{n+1 / 2}\right] \equiv \mathbf{S}_{q} \cdot \mathcal{V}_{q}^{n+1 / 2},
$$

$\mathbf{S}_{q}$ denotes the oriented surface area of the face $S_{q}$, and the nonnegative/nonpositive parts of $v_{q}^{\perp}$ always coincide with the outflow/inflow from the $P$ cell. The firstorder upwind solution is subsequently computed as

$$
\xi_{P}^{*}=\widetilde{\xi}_{P}-\frac{\delta t}{\left(\rho_{o} \vartheta\right)_{P}} \sum_{q=1}^{l(P)} F_{q}^{\perp},
$$

where $\vartheta_{P}$ is the control volume of the dual cell built about a $P$ node connected with $l(P)$ edges to its neighbours. The field $\xi^{*}$ provides the input to 
the analytically derived truncation-error compensative flux through the cell face associated with a $q$ edge evaluated as

$$
F_{q}^{* \perp}=\left|v_{q}^{\perp}\right| 0.5\left(\xi_{Q}^{*}-\xi_{P}^{*}\right)-0.5 \delta t v_{q}^{\perp}\left(\mathcal{V}_{q}^{n+1 / 2} \cdot\left\langle\rho_{o}^{-1} \nabla \xi^{*}\right\rangle_{q}\right)
$$

wherein

$$
\left\langle\rho_{o}^{-1} \nabla \xi^{*}\right\rangle_{q}=\frac{\left.\vartheta_{P} \nabla \xi^{*}\right|_{P}+\left.\vartheta_{Q} \nabla \xi^{*}\right|_{Q}}{\left(\rho_{o} \vartheta\right)_{P}+\left(\rho_{o} \vartheta\right)_{Q}}
$$

is the weighted average of the expressions evaluated in the mesh points $P$ and $Q$ connected by the edge $q$. In particular, $\left.\nabla \xi^{*}\right|_{P}$ and $\left.\nabla \xi^{*}\right|_{Q}$ represent values of spatial partial derivatives of $\xi^{*}$ calculated utilising the Gauss divergence

theorem. For example, applying the Gauss theorem to the divergence of a vector field composed of two zeros and a generic scalar field $\xi$ for the $I$ th component leads to

$$
\left.\frac{\partial \xi}{\partial x_{I}}\right|_{P}=\frac{1}{\vartheta_{P}} \sum_{q=1}^{l(P)} 0.5\left(\xi_{P}+\xi_{Q}\right) S_{q}^{I}
$$

where, $S_{q}^{I}$ denotes the $I$ th area component of the oriented surface element $\mathbf{S}_{q}$.

To assure monotonicity of the solution, $F_{q}^{* \perp}$ is suitably limited, using the limiters similar to those exposed in EQS. (24)-(26) of [51], but customised (and actually simpler) for the "infinite-gauge" option. The limited flux can be compactly written as

$$
\widehat{F_{q}^{\perp}}=\left[F_{q}^{* \perp}\right]^{+} \min \left(1, \Upsilon_{P}^{\downarrow}, \Upsilon_{Q}^{\uparrow}\right)+\left[F_{q}^{* \perp}\right]^{-} \min \left(1, \Upsilon_{P}^{\uparrow}, \Upsilon_{Q}^{\downarrow}\right)
$$

where the coefficient fields $\Upsilon^{\downarrow}$ and $\Upsilon^{\uparrow}$ correspond to the coefficients $\beta^{\downarrow}$ and $\beta^{\uparrow}$ in EQS. (25) of [51], with the exception that the cell volumes in the denominators of the latter are multiplied by the densities $\rho_{o}$ associated with the considered nodes. Even though the numerical experiments of this paper assume constant reference densities, the provided formulae are generally valid for $\rho_{o}$ varying in space.

Having (28), the transported variables $\hat{\xi}$ in (11) are calculated as

$$
\widehat{\xi}_{P}=\xi_{P}^{*}-\frac{\delta t}{\rho_{o} \vartheta_{P}} \sum_{q=1}^{l(P)} \widehat{F}_{q}
$$

\subsubsection{Solution procedure}

Granted the finite-volume discretisation in space, the entire solution procedure for (9), or in its reduced form for (1), can be now reduced to the six conceptually distinct steps. 
- Step 1: The procedure commences with the linear extrapolation of the advective mass flux components $\rho_{o} V_{I}^{n-1}$ and $\rho_{o} V_{I}^{n}$ to the $t^{n+1 / 2}$ instant at each mesh node $P$

$$
\rho_{o} V_{I}^{n+1 / 2}=1.5 \rho_{o} V_{I}^{n}-0.5 \rho_{o} V_{I}^{n-1}
$$

This provides an $\mathcal{O}\left(\delta t^{2}\right)$ estimate of the $\boldsymbol{V}^{n+1 / 2}$ argument of the NFTFV advection in (11), while assuring the solenoidality thereof, given both $\rho_{o} \mathbf{V}^{n-1}$ and $\rho_{o} \mathbf{V}^{n}$ were solenoidal.

- Step 2: Denoting the rhs forcings in (13a)-(13c) and (13d), respectively, as $\mathbf{R}^{\mathbf{V}}$ and $R^{\theta^{\prime}}$, the auxiliary variables $\widetilde{\mathbf{V}}=\left(\mathbf{V}+0.5 \delta t \mathbf{R}^{\mathbf{V}}\right)^{n}$ and $\widetilde{\theta}^{\prime}=$ $\left(\theta^{\prime}+0.5 \delta t R^{\theta^{\prime}}\right)^{n}$ that form the first argument the NFTFV advection in (11) are assembled using the preceding-time-step solutions and their respective forcings detailed in (13) but evaluated in Step 6 of the preceding time step. Analogously, for calculations using DES, the auxiliary variable $\widetilde{\nu}=\hat{\nu}+0.5 \delta t\left(2 R^{\hat{\nu}}\right)$ is assembled beforehand the conservative NFTFV solution for $\hat{\nu}^{n+1}$, in the spirit of the Euler-forward trajectory integral of (4), manifested by the factor 2 in front of $R^{\hat{\nu}}$ that marks the rhs of (4).

- Step 3: MPDATA transports the auxiliary variables $\widetilde{\mathbf{V}}$ and $\widetilde{\theta^{\prime}}$ assembled in Step 2, to provide their respective counterparts $\widehat{\mathbf{V}}$ and $\widehat{\theta}^{\prime}$ that form the explicit part of the solution in (12). For calculations with DES, MPDATA transport of $\widetilde{\nu}$ completes the prediction of $\hat{\nu}^{n+1}=\mathcal{A}\left(\widetilde{\nu}, \mathcal{V}^{n+1 / 2}, \rho_{o}\right)$.

- Step 4: A subsequent solution of the elliptic problem (19) subject to appropriate boundary conditions, leads to the update of the pressure perturbation $\varphi^{n+1}$ and its gradients which are then employed in the computations of velocity components at $n+1$. The evaluation of all partial derivatives that compose (19) relies on (27).

- Step 5: The evaluation of $V_{I}^{n+1}$ from (17) and $\theta^{\prime n+1}$ from (12d) completes the solution.

- Step 6: Finally, in preparation for Step 2 of the subsequent time step, the complete forcings $R^{\mathbf{V}}$ and $R^{\theta^{\prime}}$ are evaluated and stored, subsuming all their respective counterparts detailed in (13). As all dynamics forcings are already available at the end of Step 5, the computational effort of this step is focused on evaluating the viscous terms. In particular, the components of a discrete form of stress tensor (2) are evaluated, relying on (27) to obtain partial derivatives of the velocity components. For solutions using DES, the dynamic eddy viscosity $\mu_{t}$ is obtained from the Spalart-Allmaras model using $\hat{\nu}^{n+1}$ already predicted in Step 3 of the solution procedure. 


\section{NUMERICAL RESULTS FOR INCOMPRESSIBLE VISCOUS FLOW PAST A SPHERE}

\subsection{Preamble}

To verify the accuracy of the NFTFV solver, a simulation series of flow past a sphere at moderate Reynolds numbers is performed; hereafter Re abbreviates "Reynolds number" or "Reynolds numbers". The results are compared with both numerical and experimental data available from the literature.

Incompressible flows past a sphere at moderate Re numbers evince astoundingly complex features. Experimental studies, e.g. [68], have shown that when

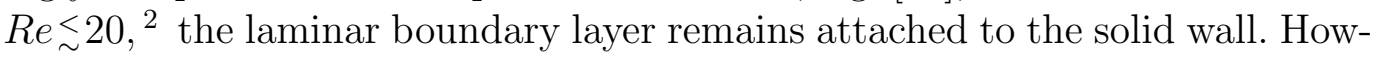
ever, as Re increases, separation occurs and the axisymmetric vortex ring is formed behind the sphere. Up to $R e \approx 210$ flow can reach steady state. For $R e \in[210,270]$, the ring becomes unstable and the vortices start loosing the symmetry. When $300<R e<420$, vortices begin to periodically shed from the sphere [39]. The position of the rolling-up vortex sheet moves closer to the sphere with increasing $R e$ [1], and the periodic shedding of hairpin vortices occurs. This regime is also known as a "regular shedding mode", and it changes to an "irregular mode" when $420<R e<800$ [40] in which the shedding direction onsets spontaneously. Approximately at $R e=800$ a high frequency Kelvin-Helmholtz instability of the separated shear layer appears, which coexists with the large scale instability in the wake due to the vortex shedding [39]. For higher Re up to about $3.7 \times 10^{5}$, the wake becomes turbulent while the boundary layer remains laminar and the values of the drag coefficient little change with Re. According to [69], the separation position stays nearly the same for $10^{4}<R e<3.5 \times 10^{5}$ while the wake undergoes periodic fluctuations. For $6000<R e<3.5 \times 10^{5}$ the Strouhal number of the vortex shedding remains almost constant, and the vortex separation point rotates around the sphere [1]. At about $R e=3.7 \times 10^{5}$ a distinct drop of drag coefficient is detected. For even higher $R e$ values, the boundary layer becomes turbulent and an attached hairpin vortex appears, behind which the streamwise line vortices are formed and then rotate slowly about the large-scale lee flow axis [68]. In the present study we selected cases for $R e=200,300$ that cover the moderate Reynolds number regime and the $R e=5000$ case to highlight a developed turbulent regime.

The dimensionless computational domain used in all simulations consists of a $20 \times 20 \times 20$ cube with a solid sphere of diameter $D=1$ located in its centre. It contains a primary tetrahedral mesh, except within a specified radial

$\overline{2}$ The precise Reynolds numbers for the outlined regimes of the flow past the sphere differ between various literature sources. 
distance from the sphere's surface where prismatic element layers are built on the basis of the triangular faces of the surface mesh. Figure 2 illustrates a typical structure of the primary mesh and shows a triangular mesh on a sphere's surface together with a fragment of the central plane cut trough the volume mesh indicating the location of triangular prisms and tetrahedral elements. The finite volume integration uses a dual mesh which is generated from such a primary mesh as demonstrated in Fig. 1.

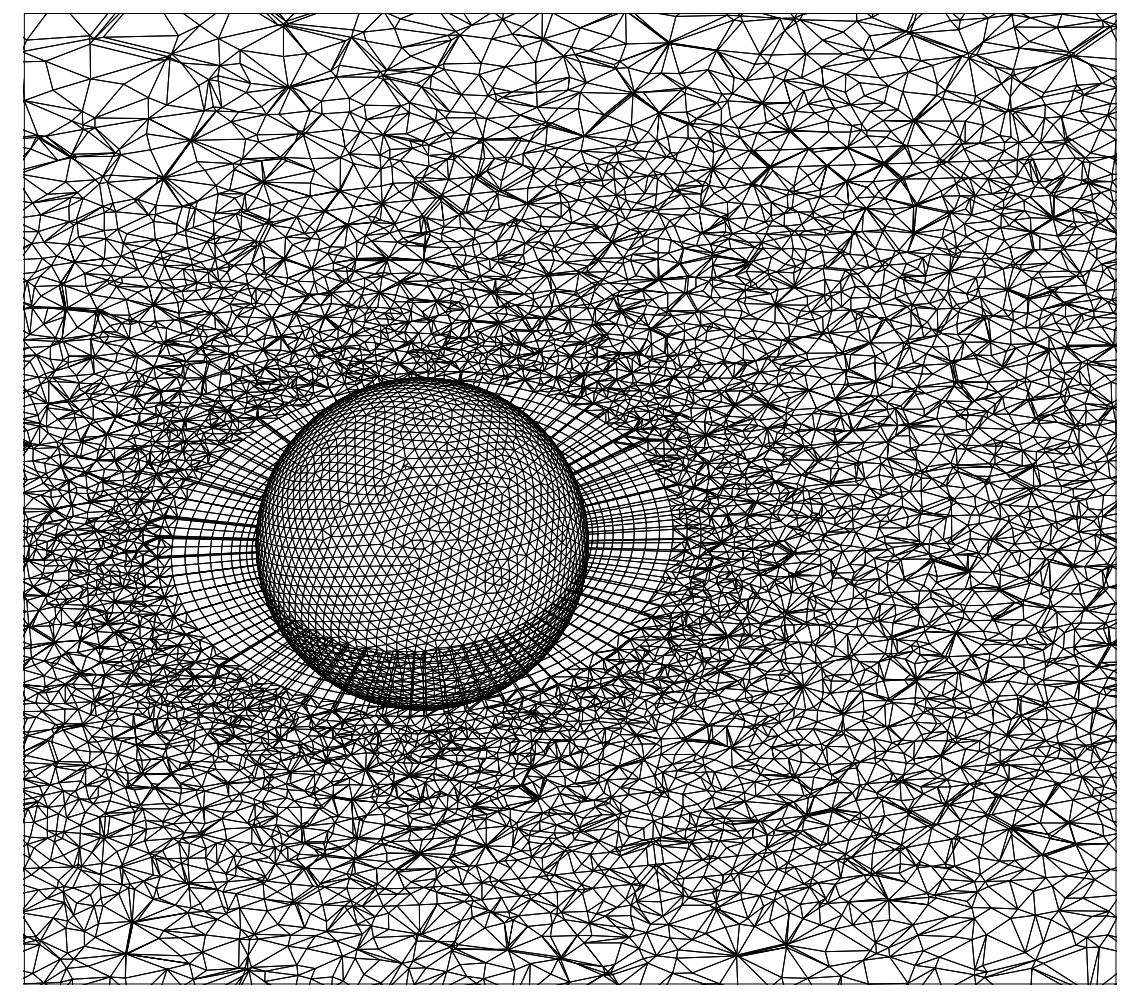

Fig. 2. A fragment of the $y=0$ cross-section of the primary mesh for flow past a sphere and the triangular surface mesh.

The sphere is placed in a fluid having a constant free stream velocity, set to $\mathbf{V}_{\mathbf{e}}=\left(V_{o}, 0,0\right)=(1,0,0)$ at the inflow and outflow. A no-slip boundary condition is imposed on the solid surface of the sphere, and the remaining boundaries are free-slip. Absorbers that attenuate the solution toward the free stream velocity components are used near the inlet and outlet, with the absorbing layers activated at the location of 2.0D away from these boundaries. The potential flow initialises the solution, and subsequently the Navier-Stokes equations (1) are solved using the NFTFV scheme.

For viscous flow past the sphere, the drag force $F_{d}$ comprises the form drag $F_{p}$ and the frictional drag $F_{f}$. In the analysis of results, the drag force acting on the sphere is integrated over the surface of the sphere $S$ and is calculated 
from

$$
F_{d}=F_{p}+F_{f}, \quad F_{p}=-\oint \rho_{o} \varphi n_{1} d S, \quad F_{f}=\oint \mu\left(\frac{\partial V_{1}}{\partial x_{J}}+\frac{\partial V_{J}}{\partial x_{1}}\right) n_{J} d S,
$$

where $d S$ indicates a boundary element (face) and $n_{I}$ the $I^{t} h$ component of the unit normal vector to the surface, and the repeating $J$ indices imply the summation. The corresponding dimensionless drag force coefficients are defined as

$$
C_{\zeta}=\frac{F_{\zeta}}{0.5 \rho_{o} V_{o}^{2} A}, \text { for } \zeta=d, p, \text { and } f
$$

where $A$ is the reference area; $A=\Pi(D / 2)^{2}$ for the sphere.

\subsection{Results and discussion for $R e=200$}

The mesh used in computations in this and the subsequent section consists of 190327 nodes. The region of prismatic elements is $0.3 D$ thick. There are 9 layers of prisms and their thickness increases in the radial direction from the sphere. The thickness of the first layer from the solid sphere is 0.014 , while the average edge length on the solid sphere is 0.03 .

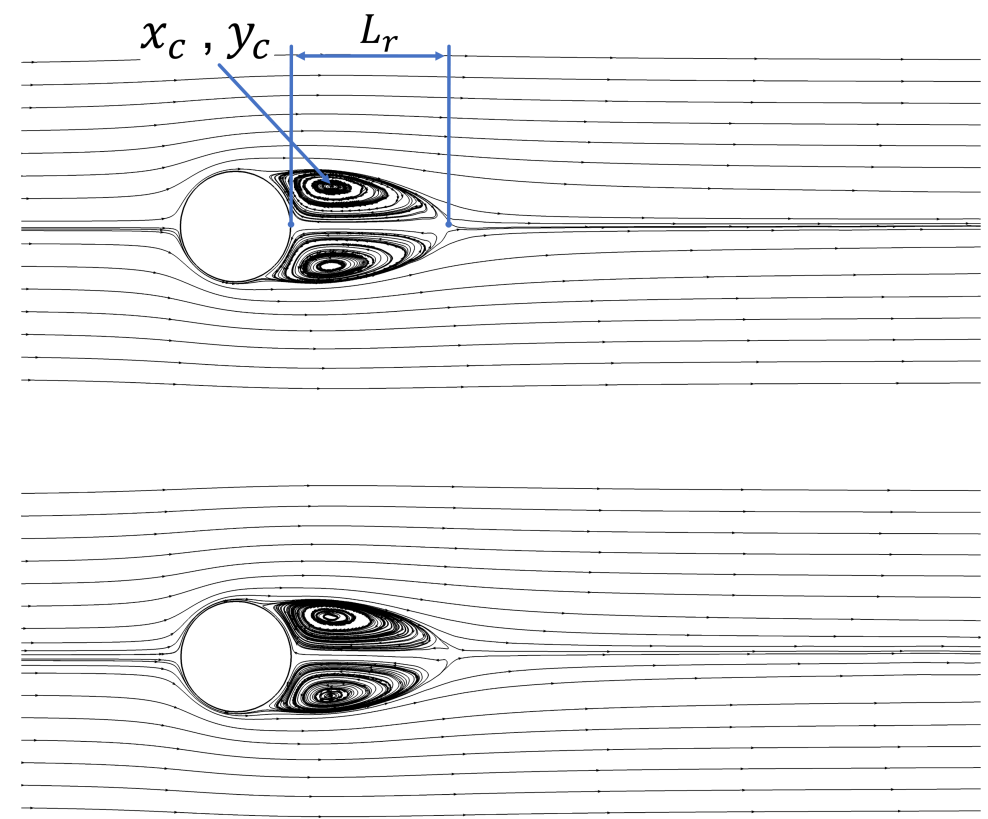

Fig. 3. Streamlines for the steady-state flow past a sphere at $\operatorname{Re}=200$, in $x-y$ (top) and $x-z$ (bottom) planes.

The top plate of Fig. 3 shows the flow streamlines in the $x-y$ plane cutting through the centre of the sphere while the bottom plate shows the streamlines 
in the $\mathrm{x}-\mathrm{z}$ plane. It can be observed that the two recirculations in the wake region of the sphere are symmetric about the centre line $(y=0, z=0)$, which is noteworthy as the streamlines are plotted based on irregularly spaced points in the unstructured mesh. The planar symmetry in these two figures implies that the flow in the wake region is axisymmetric-symmetric. This result confirms the experimental observation in [68].

Table 1

Flow past a sphere at $\mathrm{Re}=200$, comparison of experimental and numerical results for drag coefficient $C_{d}$, recirculation length ratio to diameter $L_{r}$ and separation angle $\phi_{s}$.

\begin{tabular}{cccc}
\hline \hline & $C_{d}$ & $L_{r}$ & $\phi_{s}$ \\
\hline NFTFV & 0.774 & $1.429 \mathrm{D}$ & $116.6^{\circ}$ \\
{$[34]$ (experiment) } & - & - & $116.5^{\circ}$ \\
{$[3]$} & 0.77 & $1.43 \mathrm{D}$ & $116.3^{\circ}$ \\
{$[70]$} & - & $1.429 \mathrm{D}$ & $116.1^{\circ}$ \\
{$[14]$} & 0.768 & $1.436 \mathrm{D}$ & - \\
{$[17]$} & 0.776 & $1.427 \mathrm{D}$ & $116.2^{\circ}$ \\
{$[4]$} & 0.775 & $1.430 \mathrm{D}$ & $116.7^{\circ}$ \\
{$[30]$} & 0.749 & - & $114.3^{\circ}$ \\
{$[7]$} & - & $1.436 \mathrm{D}$ & $116.3^{\circ}$ \\
{$[67]$} & 0.771 & - & - \\
{$[62]$} & 0.784 & $1.310 \mathrm{D}$ & $118^{\circ}$ \\
{$[25]$} & 0.772 & - & - \\
\hline
\end{tabular}

Table 1 documents that the computations obtained with the new NFTFV scheme are in good agreement with the earlier numerical and experimental results. Due to the irregularity of the triangular surface mesh, the separation angle $\phi_{s}=116.6^{\circ}$ was averaged from 23 points and its measured maximum and minimum values were equal to $116.8^{\circ}$ and $116.4^{\circ}$ respectively. The position of separation point on the sphere surface is found by checking the first point in the volume mesh above the sphere that has an inverse velocity gradient. The azimuthal angle is then used to describe the position separation point on the sphere surface. The recirculation bubble length was measured in both x-y plane and $\mathrm{x}-\mathrm{z}$ plane to be $1.429 \mathrm{D}$. Furthermore, the position of recirculation bubble focus which is also demonstrated in Figure 3 is at $\left(x_{c}=0.36 D, y_{c}=0.88 D\right)$. The same result is reported in [20]. Furthermore, the top plate of Fig. 8 in section 5 demonstrates the contour plots of vertical velocity component $V_{3}$ at $z=0$ plane (left panel) and $V_{2}$ at $y=0$ plane (right panel), together with corresponding in-plane velocity vectors, for the same case. It can be observed 
that both contour plots are symmetric around the centre line.

\subsection{Results and discussion for $R e=300$}

At $\mathrm{Re}=300$ the vortices shed periodically from the sphere. Figure 4 shows the history of the drag coefficient $C_{d}$ over a dimensionless time $\left(T=t V_{o} / D\right)$ from $T=250$ to 300 , and illustrates the periodic character of the vortex shedding. The drag coefficient is averaged over the same time interval, leading to $C_{d}=0.665$ with the amplitude $C_{d}^{\prime}=0.003$. This is in good agreement with [20], where $C_{d}=0.656$ and $C_{d}^{\prime}=3.5 \times 10^{-3}$ were reported for this case. Reference [36] used vortex methods and provided a slightly higher value of $C_{d}=0.683$ with $C_{d}^{\prime}=2.5 \times 10^{-3}$ while [32] reported an empirical expression for $C_{d}$ in terms of Reynolds number based on the previous experimental results, providing $C_{d}=0.654$. Overall, $C_{d}$ and $C_{d}^{\prime}$ obtained from the present study agree well with the results reported in the literature.

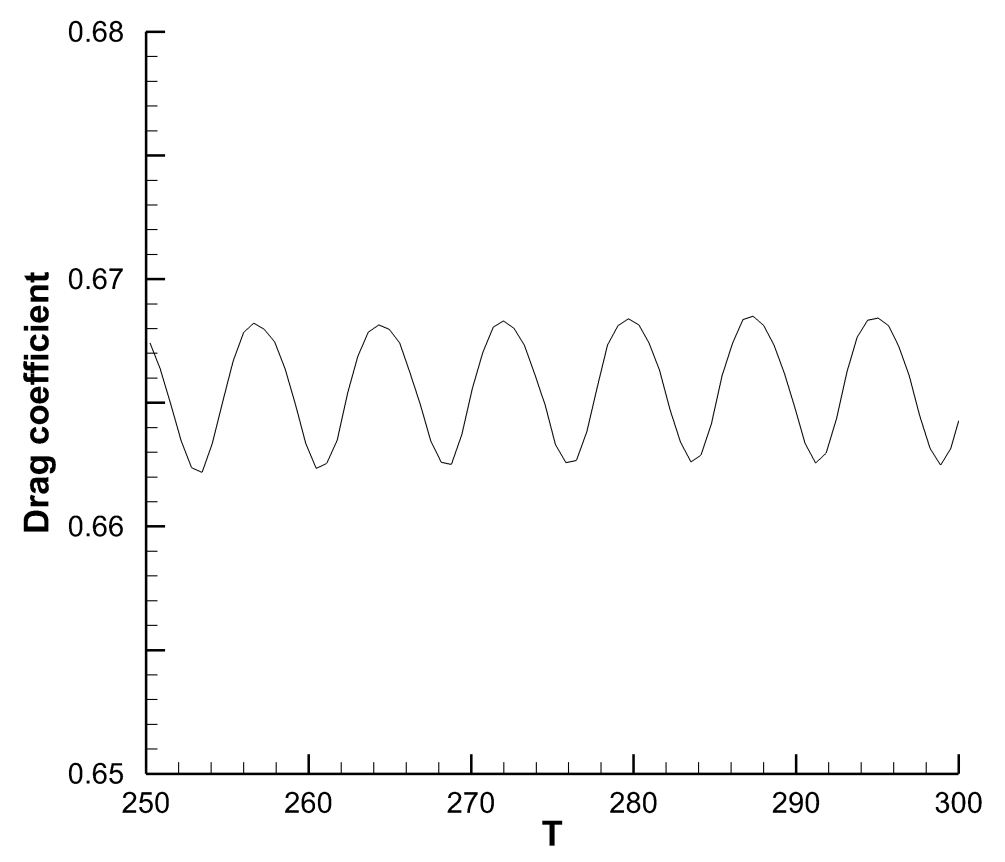

Fig. 4. The history of the $C_{d}$ drag coefficient; $\operatorname{Re}=300$.

The Strouhal number obtained from the simulation is $S_{t}=0.131$. The result given in [20] is $S_{t}=0.137$, [21] reported $S_{t}=0.133$, while [36] obtained $S_{t}=$ 0.135. Additionally, [5] reported $S_{t}=0.136$, whereas [70] obtained $S_{t}=0.136$. Based on these comparisons, the $S_{t}$ calculated in the present study is about 
$2 \%$ lower than the average $S_{t}$ from the literature - most likely due to the use of differently sized domains; cf. section 5.3 in [36] for a discussion-and all six values remain within three standard deviations of their mean.

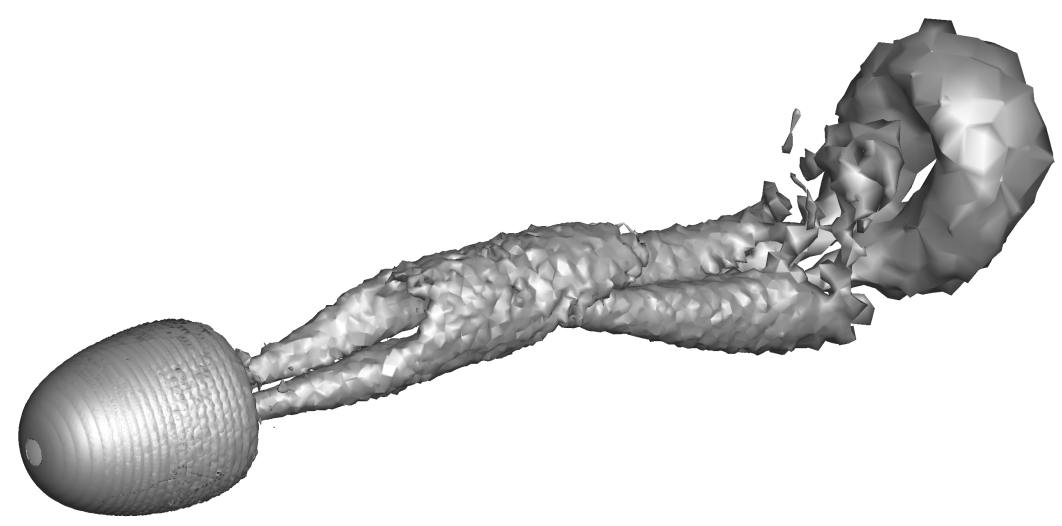

Fig. 5. A perspective visualization of the wake vortex structure for $R e=300$.

Figure 5 shows an instantaneous level set of the positive second invariant $Q$ of the deformation tensor; $Q=0.5\left(\Omega_{I J} \Omega_{I J}-\mathcal{D}_{I J} \mathcal{D}_{I J}\right)$, where $\mathcal{D}_{I J}$ is half of the tensorial part of (2), $\Omega_{I J}$ was defined in (6) and repeating indices imply the summation. This presentation is known as the Q-method for vortex identification [19]. In Fig. 5 it effectively illustrates the 3D structure of the wake flow. In particular, it evinces the structure of the hairpin vortex in the further wake region, where the mesh coarsens as approaching the outlet boundary. In the NFTFV simulation the hairpin vortex is symmetric about the central plane that corresponds to a rotated $x-z$ plane by $\approx-45^{\circ}$ about the abscissa. Importantly, this confirms the conclusion in [21] that the planar symmetry still exists when $R e=300$. The presented structure is consistent with experimental images provided in Fig. 8 of [39], and with the numerical results obtained in [20] where a further review and an extensive discussion of the flow mechanism at $R e=300$ are also provided.

\subsection{Results and discussion for $R e=5000$}

The primary hybrid mesh used in this calculation is generated using a similar strategy as in Sections 4.2 and 4.3, however, the thickness of the prismatic mesh region is now equal to the diameter $D$ of the sphere; as $R e$ increases a finer resolution is required near the solid boundary. The prismatic mesh used in this section consists of 26 layers. The thickness of the first layer from the solid sphere is 0.005 , or $y^{+}=0.98$ in wall units. ${ }^{3}$ The average size of an edge

$\overline{3}$ The $y^{+}$is calculated assuming the shear velocity $u_{\tau}=0.04 U_{\infty}$ and following the estimation of [6] with $y^{+}=y^{\prime} u_{\tau} / \nu$ and $u_{\tau}=\sqrt{\tau_{w} / \rho}$, where $y^{\prime}$ is the distance from 

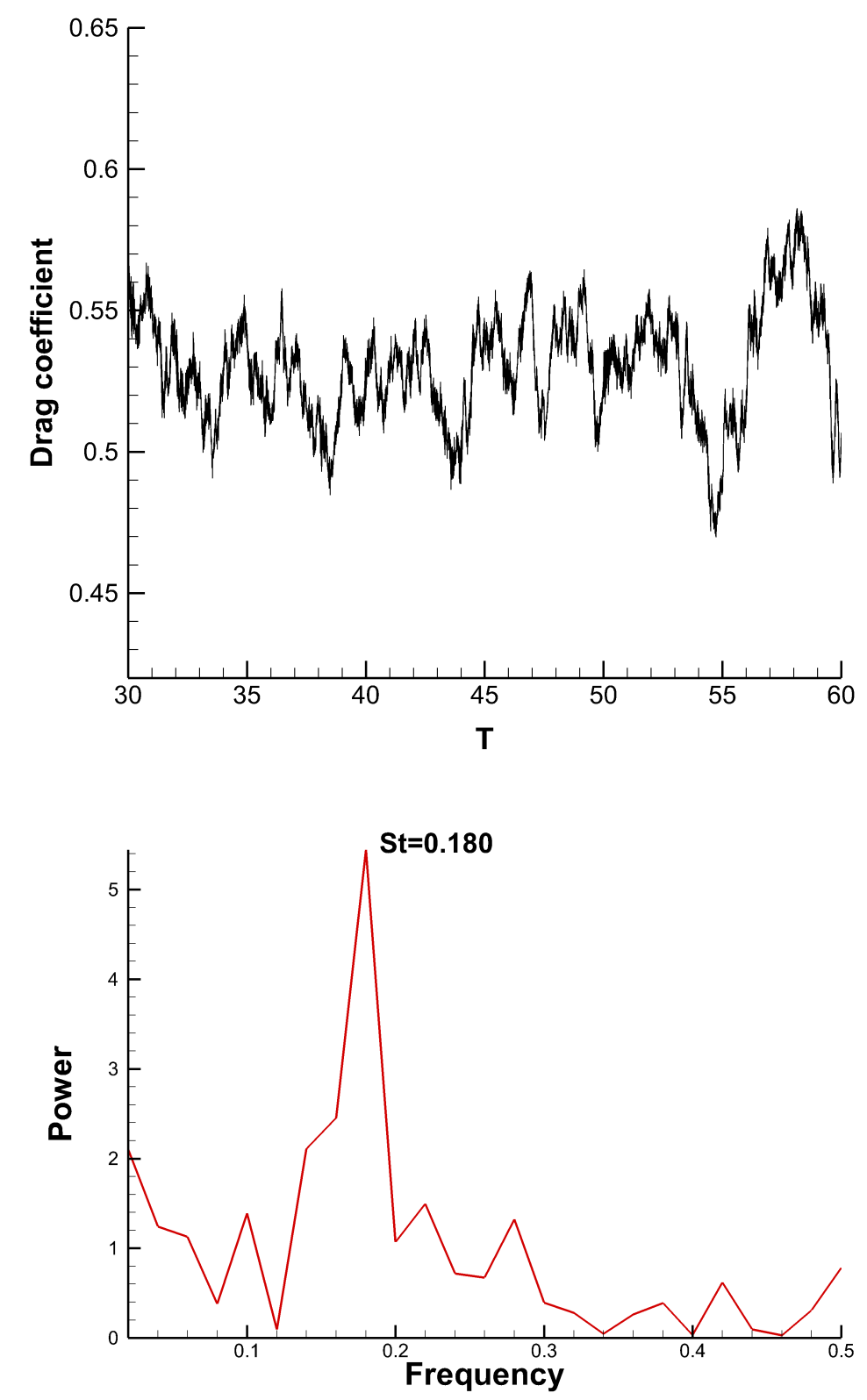

Fig. 6. The history of the $C_{d}$ drag coefficient (top) and the power spectrum (bottom); $\mathrm{Re}=5000$.

on the surface of the sphere is 0.02 and the total number of points in the mesh is 777506 points.

The NFTFV solution of the Navier-Stokes equations (1) with the DES option was used in this test case. The resulting history of drag coefficient for the non-

$\overline{\text { the wall }}$ and $\tau_{w}$ is the wall shear stress. 
dimensional time interval from $T=30$ to 60 and the corresponding power spectrum are shown in Fig. 6. The mean drag coefficient $C_{d}=0.527$ was obtained by taking a time-average value over the interval. The upper panel of Fig. 6 shows that the oscillation frequency of $C_{d}$ is now much higher than that for the case with $R e=300$. This frequency is mainly due to the occurrence of the instability in the detached shear layer [6]. The value $C_{d}=0.468$ was obtained from the LES calculation for $R e=5000$ in [16]. The value $C_{d}=$ 0.38 was obtained using DNS in [41], and $C_{d}=0.393$ using DES in [7]. An experimental result from [29] is $C_{d}=0.52$. Overall, the value of $C_{d}$ obtained from the NFTFV scheme is higher than the cited numerical results, but is reasonably close to the experimental result in [29], although the latter one includes a small effect of a thread used in the experiment to suspend a sphere, cf. their Fig. 3. We also note that there is some influence of the irregularity of the unstructured surface mesh in our analysis of the drag coefficient. The frequency of $C_{d}$ shown in the bottom panel of Fig. 6 indicates that the mean value from the dominant frequency obtained from the NFTFV simulation agrees well with the numerical and experimental results $[23,7,6,16,35]$.

The separation angle of the laminar boundary layer is determined by the forces acting on the surface of the sphere. The NFTFV scheme using DES predicts $\theta_{s}=\left[88^{\circ}, 89^{\circ}\right]$, which is in good agreement with the literature; e.g. [41] reports $\theta_{s}=89.5^{\circ}$ while $[38]$ reported $\theta_{s}=89^{\circ}$.

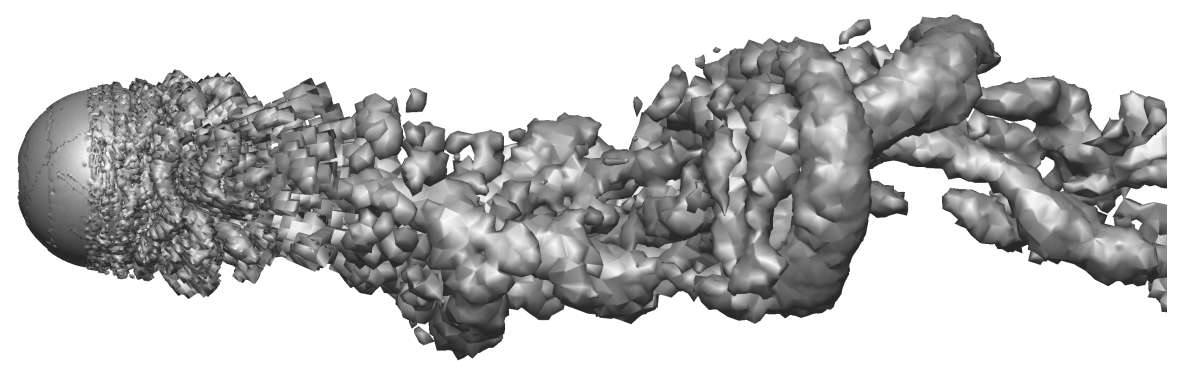

Fig. 7. A perspective visualisation of the wake vortex structure at $R e=5000$.

Similar to Fig. 5, Fig. 7 shows the 3D structure of the wake flow at one order of magnitude larger $R e=5000$. In contrast to the $R e=300$ case, a large number of vortex rings with a range of scales and irregular rotation can be observed in the wake region. This indicates a higher frequency of the vortex shedding mode and a more complex interaction between different scales of vortexes in the near 
wake than at $R e=300$. Additionally, it can be seen that the large-scale vortex consists of several smaller scale vortex rings, which are topologically similar to the finding in [38]. A closer examination of the shedding of vortex tubes on the sphere's surface shows that the detached shear layer breaks down into rings just after the separation point. The rings then travel downstream and form vortex tubes behind the sphere. A similar vortex tubes formation is documented in [23]. This pattern also agrees well with the experimental result in [39] for the transitional region $\mathrm{C}\left(3 \times 10^{3}<R e<6 \times 10^{3}\right)$ specified and illustrated in their Table 1 and Fig. 8d, respectively.

\section{STRATIFIED LAMINAR FLOW PAST A SPHERE}

\subsection{Motivation}

Numerical models for global flows are the staple of computational meteorology. However, from the perspective of numerical designs there is an essential difference between the atmospheric models for global weather and climate and the models for external flows past a sphere discussed in this paper. The former are posed in a thin spherical shell with radial extent small compared to the radius of the sphere [64,57], whereas the latter are formulated in a Cartesian domain of a substantially larger extent than the radius of a sphere. These latter models bear a resemblance to mesoscale atmospheric models for simulating orographic flows $[56,66]$ with the exception of the disparity of scales. The vertical extent of natural mountains and volcanic islands is sufficiently large to make relevant the effects due to the vertical variability of physical properties of the atmosphere, and this atmospheric stratification adds another dimension to the problem of viscous and inviscid flows past immersed bodies.

For illustration, consider that for an inviscid adiabatic flow in a stably stratified atmosphere the momentum equation (9b) can be written in a dimensionless form

$$
\frac{d \mathbf{V}}{d t}=-\nabla \psi-\frac{\eta}{F r^{2}} \mathbf{k},
$$

where $\mathbf{V}$ and $\psi$ mark the dimensionless velocity vector and pressure perturbation, respectively, while $\eta$ denotes the displacement of a fluid parcel from its datum $z_{0}$ far upstream of the body immersed in a uniform ambient flow $\mathbf{V}_{o}=\left[V_{o}, 0,0\right] ; \mathbf{k} \equiv \nabla x_{3}$ is the vertical unit vector. ${ }^{4}$ The equation (33) hints

\footnotetext{
$\overline{4}$ Note that $d \theta / d t=0$ implies $\theta(\mathbf{x}, t)=\theta_{e}\left(z_{o}\right) \equiv \theta_{e}\left(x_{3}-\eta\right)$, so expanding the latter in the first order Taylor series about $\eta=0$ reveals the buoyancy in terms of $\eta$.
} 
that variation of flow realisations for $\operatorname{Fr} \in[0, \infty]$ must be intricate. Multiplying (33) by $F r^{2}$ and taking the limit $F r \searrow 0$ implies $\eta \equiv 0$, i.e. flat isentropes and a 2D potential flow around the body. On the other hand, taking in (33) the $\operatorname{Fr} \nearrow \infty$ limit implies vanishing buoyancy and thus a 3D potential flow. In effect, the stated problem cannot be properly posed (cf. §III.6.2 in [8]), as at some $\mathrm{Fr}$ there must be a discontinuous transition from simply to multiply connected isentropes, comprising holes through which the body protrudes. Furthermore, if an inviscid flow transitions from large to small Froude numbers in a finite-time [9], the connectivity of the material surfaces must not change, and if it does than some form of viscosity must be involved [33]. Still, inviscid linear $[43,44]$ and asymptotic [11] theories provide insights into flows at, respectively, large and small $F r$. While the linear theory is quite powerful in providing meaningful predictions in terms of $\mathrm{Fr}$ for the upwind stagnation and the associated phenomena of flow reversal and upwind blocking, the asymptotic theory merely hints the tendency for the fluid to pail up on the the lower upwind side and solution approaching the horizontal potential flow in the limit of vanishing Fr; see [48] for a discussion. Neither of the two theories captures the flow morphology in the range of $0 \lesssim F r \lesssim 0.5$ where the flow evinces pronounced vortical structures in the horizontal and vertical (horseshoe, cowhorn and lee eddies) observed in nature, laboratory, and numerical simulations; cf. $[18,46,47,71,12]$. In the lack of discriminating measurements or theory, there is a long-lasting controversy on the relative roles of viscosity and stratification in generating the intricate solutions throughout the high to low $\mathrm{Fr}$ regimes; cf. [71,12] for discussions.

The results of the preceding section bolster our confidence in the integrity of the NFTFV solvers, and the approach appears well-positioned to address viscous stratified flows past sphere. In the context of the above-mentioned controversy, the sphere is especially attractive because there are published results of earlier pertinent laboratory and numerical studies that have been inaccessible for decades to the state-of-the-art atmospheric models, predominantly based on structured grid discretisations of simply connected domains. The past experimental and numerical studies include [29,26,15]. Experiments [29] measured the drag on a sphere moving horizontally through stratified fluid, over a wide range of Reynolds and Froude numbers. These experiments provided quantitative data for corresponding drag coefficients. Moreover, [26] provides visualisations from experiments for $\operatorname{Fr} \in[0.005,20]$ and $R e \in[5,10000]$ showing a range of characteristic flow phenomena. Numerical investigations in [15] focused on stratified flows at $R e=200$ and employed a structured grid that extended in the radial direction from a sphere. In the following subsection, we repeat simulations proposed in [15] but use a hybrid mesh providing relatively high spatial resolution both close to the sphere surface and in the wake. 


\subsection{Flow patterns for $\mathrm{Fr} \nearrow \infty, 1$ and 0.25}

The construction of computational mesh, problem domain and boundary conditions are essentially the same as in Section 4.2. However, the distance between the first layer of points off the sphere is decreased to 0.0096 and there are 24 prismatic layers. The mesh consists of 770331 points and the average edge length on the sphere is 0.02 . As before, the sphere is placed in a constant flow $\mathbf{V}_{\mathbf{e}}=\left(V_{o}, 0,0\right)=(1,0,0) \mathrm{ms}^{-1}$ and the kinematic viscosity $\nu=0.005 \mathrm{~m}^{2} \mathrm{~s}^{-1}$ is chosen to give $R e=200$, fixed through all calculations. Since both $V_{o}$ and the diameter of the sphere $D$ are constant, the Froude number $F r=2 V_{o} / N D$ is changed between $F r \in[0.25,200]$ by varying the buoyancy frequency $N=\left(g S_{o}\right)^{1 / 2}$; recall that stratification $S_{o}$ was introduced at the occasion of (9). ${ }^{5}$ Computations for a range of $F r$ were carried out using the procedure outlined in section 3.3 until flow patterns have been established. This required a simulation over the non-dimensional time $T=t V_{o} / D=30$ to allow the flow to travel a distance of $30 D$.

The simulated flow patterns are illustrated in Fig. 8 that displays the relevant velocity contours and vectors on the central vertical $\left(y \equiv x_{2}=0\right)$ and horizontal $\left(z \equiv x_{3}=0\right)$ cross-sections. ${ }^{6}$ All contour plots use 0.1 interval with the maximum and minimum contour values set at $\pm 0.5 \mathrm{~ms}^{-1}$ respectively and the dashed lines indicating negative values. The displayed patterns compare well with Fig. 3 in [15]. In particular, the top row of Fig. 8, for $\operatorname{Fr} \nearrow \infty$, represents a limit where the flow becomes neutrally stratified, hence reduced to a laminar flow past a sphere at $R e=200$. As discussed in section 4.2 , the results show a formation of a steady axisymmetric re-circulation bubble behind the sphere with the flow near the central line $(y=z=0)$ being advected back towards the sphere. The fluid parcels symmetrically flow around and over the sphere. The contour plots, streamline patterns, and the velocity vectors on both $y=0$ and $z=0$ planes are almost identical. Since the eddy is axisymmetric, planes $y=0$ and $z=0$ divide the lee-side flow into four identical regions. The flow patterns for Froude number $F r=200$ (not shown), remain almost unchanged from those depicted for the neutrally stratified flow.

At $F r=1$, the central row of Fig. 8 demonstrates that the flow is very different from the neutral case. Clearly, the symmetry is broken between the horizontal and the vertical flow pattern. This is due to the buoyancy-induced gravity waves radiating in the lee, and the resulting 3D structure of the wake. The $F r=1$ case is special, as for the inviscid flows crude estimations based on

\footnotetext{
5 Because $V_{3}=d \eta / d t,(33)$ reveals the buoyancy in a stably stratified fluids as a restoring force with oscillation frequency proportional to $N$.

6 The velocity vector plots are shown after mapping the results onto a relatively coarse Cartesian mesh
} 

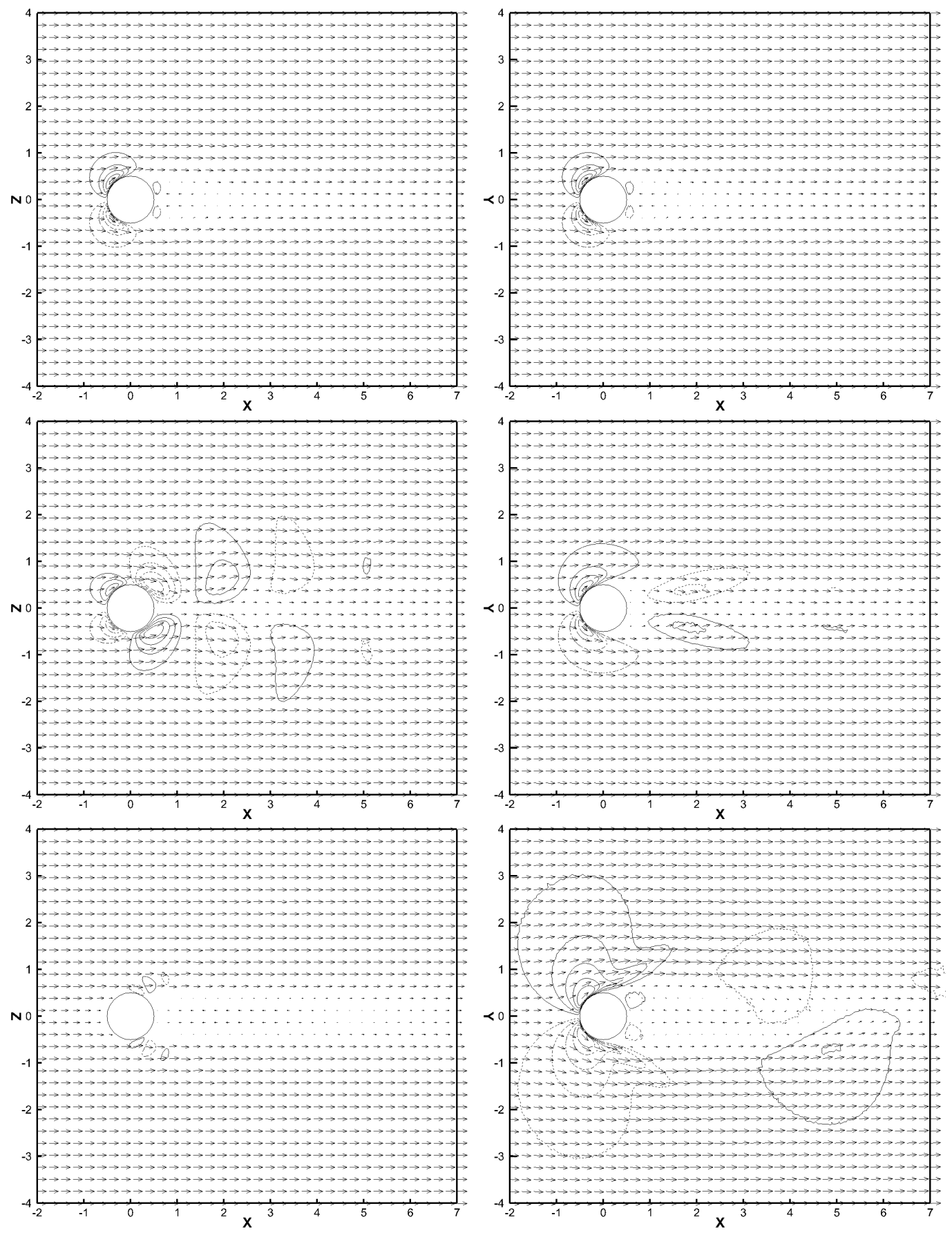

Fig. 8. Contour plots of vertical velocity component $V_{3}$ at $y=0$ plane (left) and a transverse velocity component $V_{2}$ at $z=0$ plane (right) together with velocity vectors for stratified flow past a sphere at $\operatorname{Re}=200$; top $\operatorname{Fr} \nearrow \infty$, centre $F r=1$ and bottom $\mathrm{Fr}=0.25$.

the Bernoulli equation [45] predict the incipient stagnation at the obstacle top-leading to the concept of the dividing streamline height $h_{s}=h(1-F r)$ implying flow trajectories with datums $z_{o}<h_{s}$ to impact on the obstacle surface, while going over the top otherwise [18]. However, refined linear theory $[43,44]$ past smooth hills with gentle slopes $\approx 0.1$ and the accordant numerical 
simulations [48] show this to occur at about twice smaller Fr. Noteworthy, the dividing-streamline idea has been tested in the laboratory experiments for smooth hills with slopes $\approx 1$ [18], and illustrated by means of numerical simulation for $\mathrm{Fr}=0.33$ in [66]. In case the concept of the dividing streamline applies literally, its one consequence is that the lee-wave amplitude approaches its maximum at $F r=1$, because for smaller $F r$ the lee waves are excited by effectively lower obstacles with $h_{\text {eff }}=h F r$; cf. Figs. 7 and 8 in [66] for illustration.

At $F r=0.25$, the bottom row of Fig. 8 shows that the vertical wave motion becomes weaker, while the horizontal eddies become larger. Qualitatively, this is due to sixteenfold stronger stratification compared to $\mathrm{Fr}=1$ case, upon which the majority of the mass flow is confined below $h_{s} \approx 0.75(D / 2)$ and diverted around the sphere. More quantitatively, the linear theory estimates the vertical wavelength of the stationary wave radiated aloft as $\lambda \sim \Pi F r D$. This is consistent with $\lambda \approx 3 D$ measured in the left panel of the central row of Fig. 8 and $\lambda \approx 0.7 D$ measured in the left panel of the bottom row. Consequently, a meagerly looking gravity wave at $F r=0.25$ is actually well resolved in vicinity of the sphere. Its about fourfold weaker amplitude than in the $F r=1$ case can be partially explained by the diminished mean slope of the effective obstacle above the dividing streamline, that roughly scales as $[\mathrm{Fr} /(2-$ $F r)]^{1 / 2}$ as $F r$ decreases from unity to zero. As this only accounts for about half of the difference, the other half is due to the dissipation. Indeed, on the scale of the gravity wavelength $\lambda=0.7 D$ the dissipative effects are significant as the dimensionless e-folding time scale of the dissipative attenuation of the dominant wavenumber $k=[\operatorname{Fr}(D / 2)]^{-1}$ mode is $\tau V o / D \sim 0.25 F^{2} \operatorname{Re} \approx 3$, i.e. threefold of the time scale of advection and the tenth of the duration of the experiment. As all processes are well resolved in the vicinity of the sphere, and all examined flow patterns compare well with those in [15], it is meaningful to quantify the relative importance of viscosity and stratification.

\subsection{Quantification of relative effects due to stratification and viscosity}

Following $[29,15]$, the impact of stratification on a viscous flow past a sphere is measured with the departure $\Delta C_{d}(R e, 1 / F r)$ of the drag coefficient at $1 / F r>$ 0 relative to the reference value $C_{d}(R e, 0)$ for the neutrally stratified flow

$$
\Delta C_{d}=C_{d}(R e, 1 / F r)-C_{d}(R e, 0) .
$$

Figure 9 -inspired by Fig. 4 in [29] - documents the variation of $\Delta C_{d}$ in function of $1 / F r$ for $R e=200$. The numerical NFTFV and [15] solutions (shown with the solid and dashed line, respectively) were both computed for $R e=200$. However, the laboratory experiments were conducted in series with fixed values of $N \propto R e / F r$ but varying $V_{o}$, and so with varying $F r$ and $R e$. All the labo- 


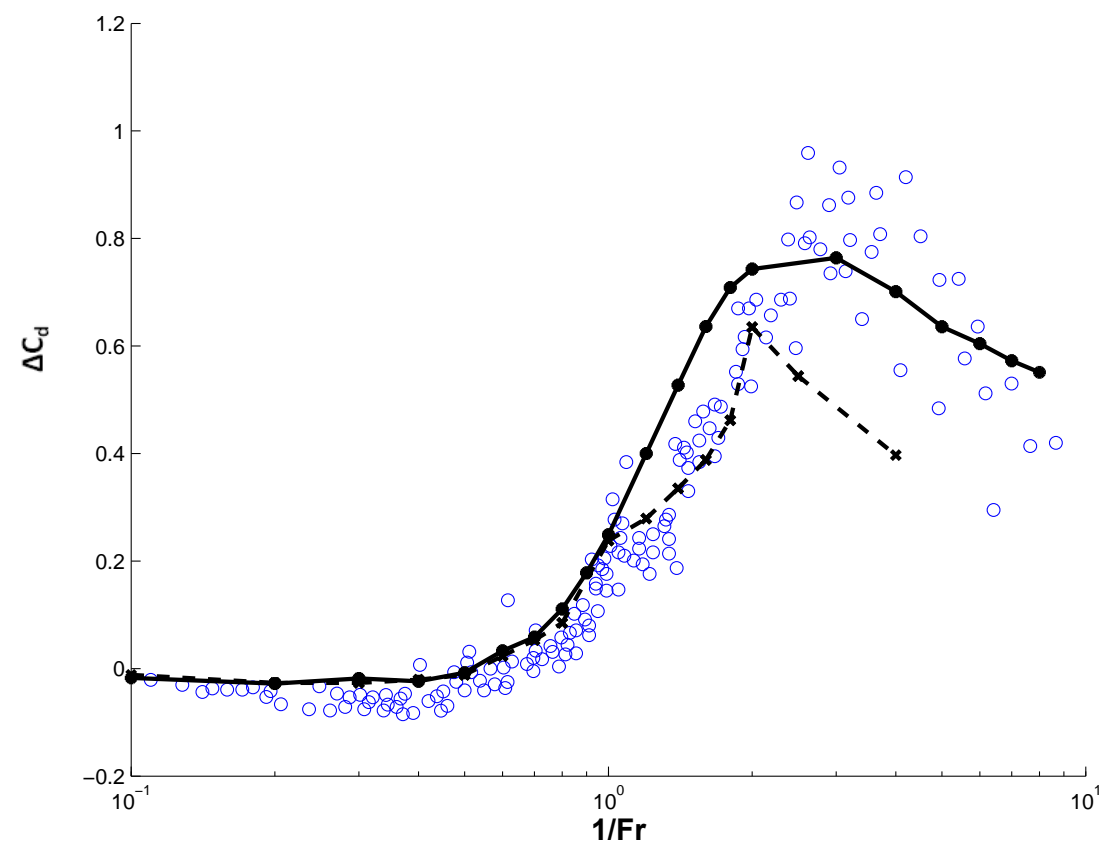

Fig. 9. $\Delta C_{d}$ in function of the inverse Froude number $1 / F r$ for numerical solutions at $R e=200$, NFTFV (solid line) and [15] (dashed line) as well as for the experimental data [29] (circles) at variable $R e \in\left[10^{2}, 10^{4}\right]$.

ratory data (marked with circles in Fig. 9) were obtained for $R e \in\left[10^{2}, 10^{4}\right]$, with the majority for $1 / F r \in[0.5,2]$ with $R e>1000$.

Figure 9 shows that for $1 / F r \in[0.1,0.5], \Delta C_{d}$ is close to zero, implying that the impact of weak stratification on the drag coefficient $C_{d}$ (defined in EQ. 32) is insignificant. For $1 / F r \in[0.5,2], \Delta C_{d}$ increases considerably, roughly doubling the Table 1 values. At $1 / F r=1.0$, the slope of the numerical solution [15] decreases sharply (perhaps too sharply in our paraphrase of the original Fig. 8 in [15]) and then continuously increases matching reasonably the laboratory data read from Fig. 4 in [29] in the range $1 / F r \in[0,2]$. However, the slope of the solid line for the NFTFV result changes little until $1 / F r=2$, and only then is followed by a sharp decrease; see also $\S 6$ in [71] for a discussion. Interestingly, the sharp transition at $F r=1$ emphasises the dividing streamline concept of [18], whereas a smooth transition through $F r=1$ with the subsequent regime change at $F r \approx 0.5$ is consistent with the linear theory of $[43,44]$ and the numerical/laboratory results in $[48,71]$. The measured heights of the dividing streamline summarized in Fig 30 of [26] show that crude energetic estimations of [45] only verify for $F r<0.7$, otherwise being overwhelmed by viscous boundary layer effects (sensitive to $R e$ ) near the obstacle base. Overall, the agreement of the NFTFV results with the experimental data of [29] is quite reasonable in the entire range of the Froude numbers $1 / F r \in[0.1,8]$ even though the laboratory experiments were mostly conducted at substantially larger $R e$. The latter is consequential, because the sharpness of the above-discussed transition at $F r=1$ appears to diminish with decreasing $R e$, 


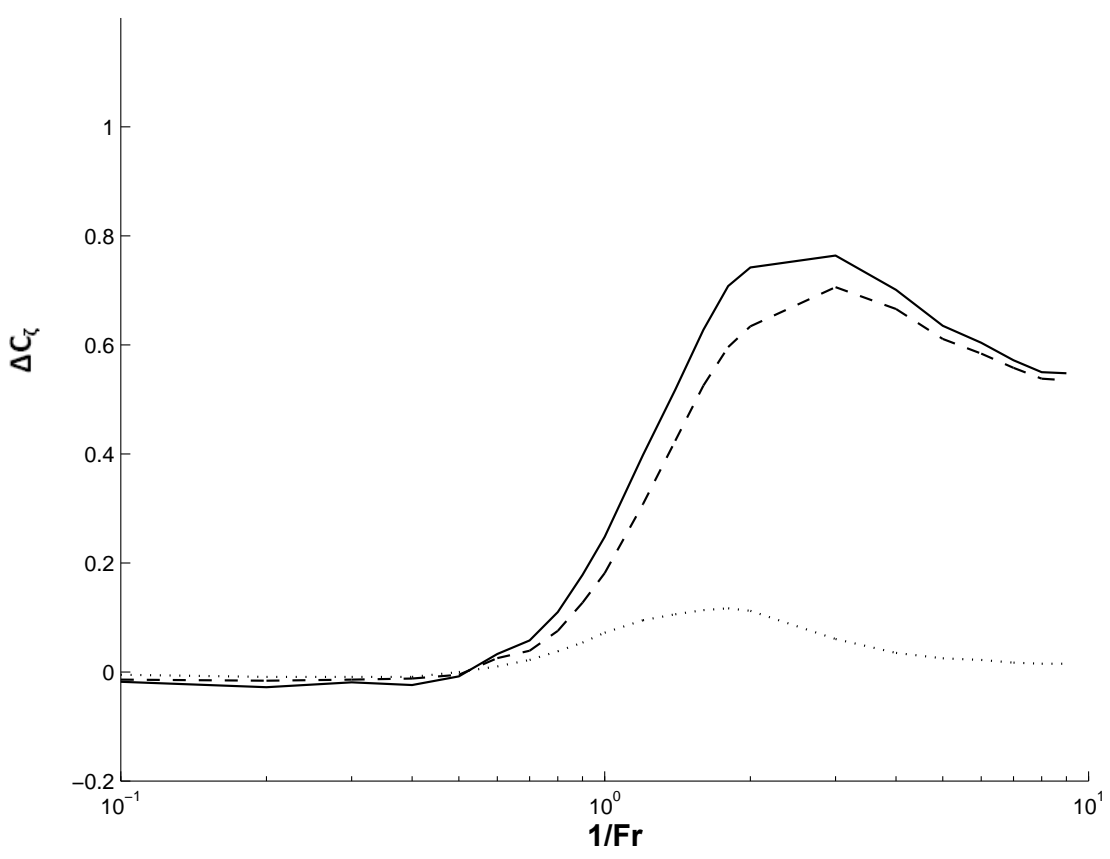

Fig. 10. NFTFV results at $R e=200$, change of $\Delta C_{d}$ (solid line), $\Delta C_{p}$ (dashed line) and $\Delta C_{f}$ (dotted line) as $1 / F r$ increases from 0.1 to 8.0.

as indicated by Figs. 3 and 4 of [29]. Notably, the numerical results of [15] poorly capture the measured values of $\Delta C_{d}$ for $1 / F r>2$ when the flow is strongly stratified and can be spectacularly intricate [66].

Figure 10 shows the total drag departure defined by (34) in function of the inverse Froude number for $R e=200$, and its breakdown into viscous $\left(\Delta C_{f}\right)$ and the form drag $\left(\Delta C_{p}\right)$ components, defined accordingly as

$$
\begin{aligned}
\Delta C_{p} & =C_{p}(\operatorname{Re}, 1 / F r)-C_{p}(\operatorname{Re}, 0), \\
\Delta C_{f} & =C_{f}(\operatorname{Re}, 1 / F r)-C_{f}(\operatorname{Re}, 0)
\end{aligned}
$$

with the drag coefficients $C_{p}, C_{f}$ defined in (32). The values of $\Delta C_{f}$ are generally small compared to $\Delta C_{p}$, and equally small compared the neutral flow values listed in Table 1 . The variability of all three $\Delta C_{\zeta}$ with $1 / F r$ values is well correlated up to $1 / F r \leq 2$, while for $1 / F r>2$ the decrease of $\Delta C_{f}$ precedes that of $\Delta C_{p}$. The magnitude of $\Delta C_{f}$ is close to the analogous result in Fig. 9 in [15], and the difference in $\Delta C_{d}$ comes mainly from $C_{p}$. The differences in pressure distribution can also be assessed by comparing distribution of NFTFV $\varphi$ around the sphere with Fig. 4 in [15]. For Froude number $F r=200,2$ and 1, these distributions (not shown) compare well with each other, and this is also where the values of $\Delta C_{d}$ in the two studies agree. For Froude number $F r=0.7,0.5$ and 0.25 , the $\varphi$ distributions show considerable departure from each other, and this is also where the values of $\Delta C_{d}$ disagree.

Altogether, the results shown in the paper strongly suggest that for natural 

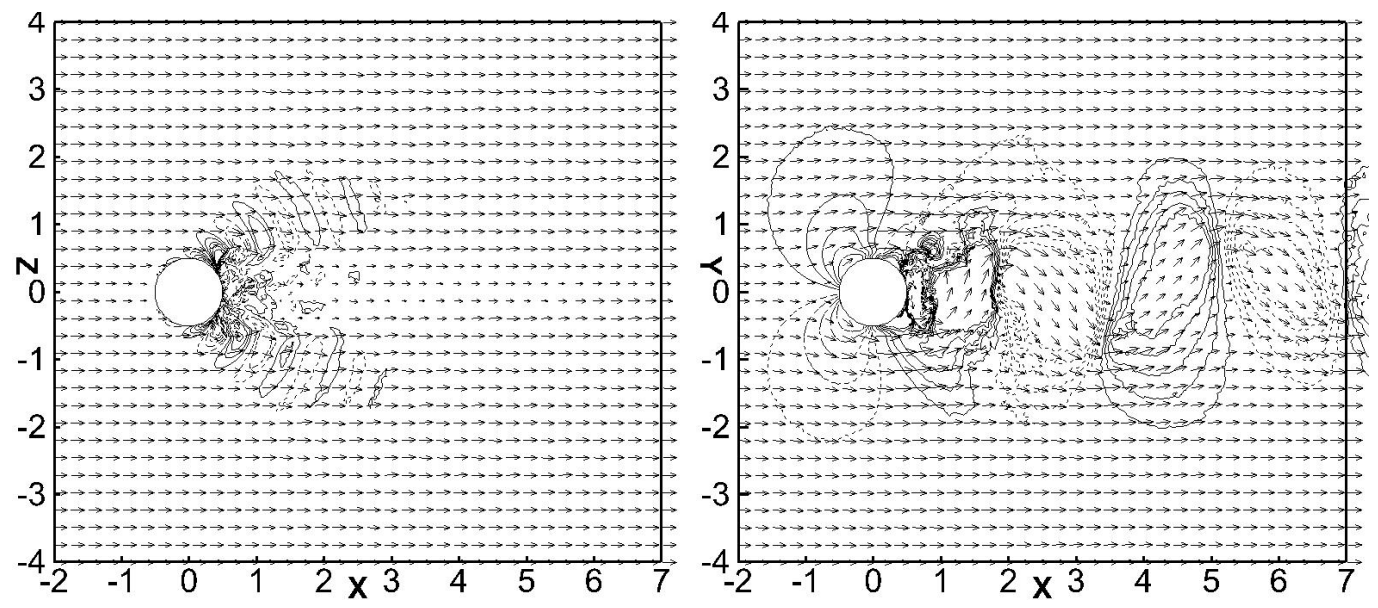

Fig. 11. Contour plots of vertical velocity component $V_{3}$ at $y=0$ plane (left) and a transverse velocity component $V_{2}$ at $z=0$ plane (right) together with velocity vectors for stratified flow past a sphere at $\operatorname{Re} \nearrow \infty$ and $F r=0.25$.

atmospheric/oceanic flows it is the stratification that controls the flow, and that viscosity mostly diminishes the amplitude of, and smears, the buoyantly induced perturbations. To amplify, Fig. 11 shows the flow pattern for implicit large-eddy simulation (ILES) of the strongly stratified flow with zero explicit viscosity and $F r=0.25$. While the solution evinces small scale features, the large aspects of the flow organization are similar to those in the bottom row of Fig. 8 for the same $F r$ and $R e=200$. Clearly the viscosity plays the role in the latter, however the former develops in the absence of the solution independent viscosity. The dissipation in ILES is flow dependent and confined to the scales comparable with the mesh resolution, just like intermittent viscosity of explicit subgrid-scale models for high Reynolds number flows $[10,37,55,57]$. It might be argued that such a viscosity determines the flow realisation. On the other hand, Fig. 12 attests that in the absence of stratification, the NFTFV solver maintains the potential flow-initiated from the uniform ambient state $\mathbf{V}_{\mathbf{e}}=$ $\left(V_{o}, 0,0\right)=(1,0,0) \mathrm{ms}^{-1}$ - over a long simulation time with high fidelity, as measured by the drag coefficient $C_{d}=2.03 \times 10^{-5}$ averaged over the $T=$ $20 \div 30$ interval. This implies that even if the viscosity plays a role in the incipient stagnation or flow reversal, it must do so in response to the flow induced by buoyant perturbations.

\section{CONCLUDING REMARKS}

The main contribution of this paper is a new numerical approach for accurate simulation of viscous, neutrally and stably stratified flows at moderate Reynolds numbers. The MPDATA-based non-oscillatory forward-in-time finite-volume (NFTFV) schemes were originated over decade ago [50,51] and have been since advanced to provide simulation tools for high Reynolds num- 


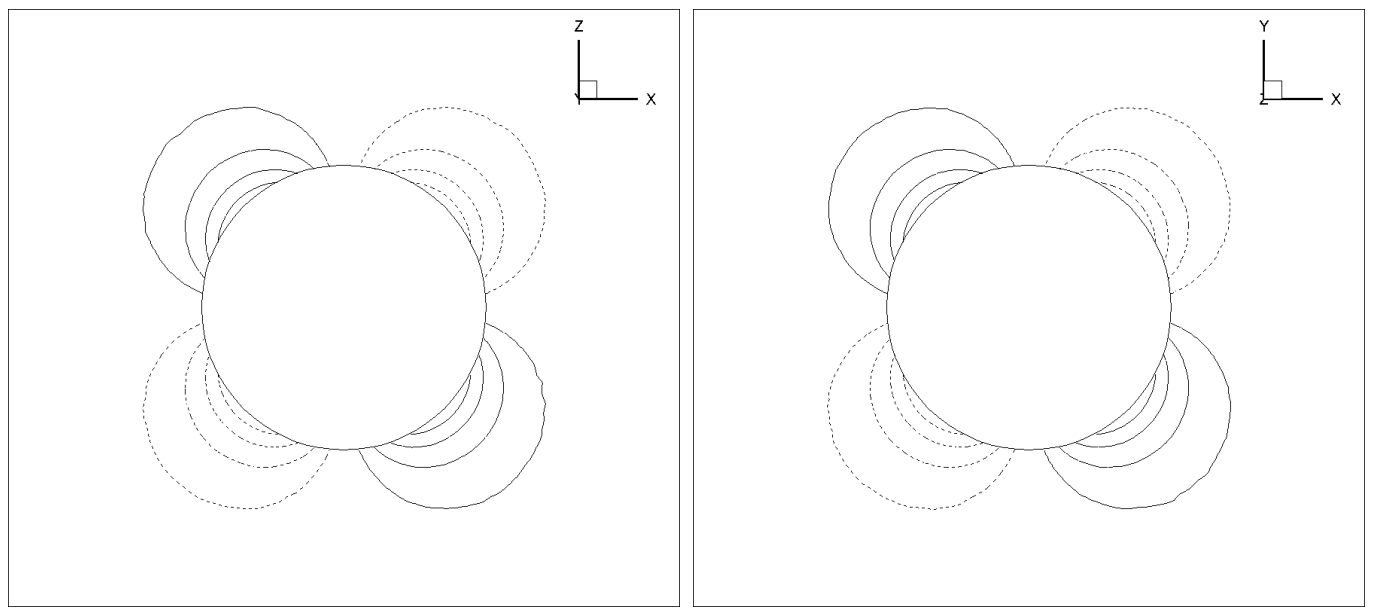

Fig. 12. Potential flow solution for $\mathrm{T}=30$. Contour plots of vertical velocity component $V_{3}$ at $y=0$ plane (left) and a transverse velocity component $V_{2}$ at $z=0$ plane (right); the contour interval and extrema are 0.5 and $\pm 2 \mathrm{~ms}^{-1}$, respectively, with dashed lines indicating negative values.

ber flows in predominantly geophysical applications. The proven implicit largeeddy simulation (ILES) capability of the MPDATA has been particularly enabling for these advancements; cf. [58] for a recent review. Yet, because ILES fundamentally relies on dissipative properties of the leading truncation terms of non-oscillatory advection, it lacks the implicit filtering action in a no-flow direction, and especially in the direction normal to an impermeable boundary. To alleviate this drawback, the ILES codes can be supplied with remedies in some form of subgrid-scale turbulence models or, in particular, wall models near solid boundaries [56]. In contrast, the presented NFTFV approach to the Navier-Stokes equations accounts for viscous effects using the complete stress tensor. This extension of the NFTFV schemes enables a broad range of simulation strategies from direct, to detached-eddy and explicit large-eddy simulations.

Generally, the semi-implicit time-stepping is beneficial both in terms of accuracy and computational economy, but it can lead to cumbersome implementations. However the collocated data arrangement makes the presented herein semi-implicit integrators simple to implement. In many schemes data collocation is notorious for supporting nontrivial null spaces of the discrete differential operators and needs to be judiciously stabilised. In the NFTFV schemes for high Reynolds number flows such stabilisation is not required, thanks to the ILES properties of MPDATA. However, this benefit may not extend to flows with the characteristic time scales of the physical forcings comparable to, or much shorter than, the time scale of advection. An important practical aspect of this paper, is the demonstration that the MPDATA-based NFTFV semiimplicit integrators provide adequate solution regularisation at the limit of the mesh resolution, but do not dilute the resolved scales. In effect, this regularisation seamlessly complements genuine collocated median-dual unstructured 
discretisation of the viscous terms at a range of the Reynolds numbers. This shows that the inherent dissipation of the NFTFV solver does not add but adapts to the physical dissipation present in the system.

The presented extension of the NFTFV integrators to incompressible viscous flows at moderate Reynolds numbers utilises topological flexibility of the finite volume discretisation on unstructured/hybrid meshes to enable a systematic study of flows past a sphere. Such flows have been studied extensively, both numerically and experimentally, in that they provide a representative range of benchmarks for external flows past immersed obstacles. Technical developments presented in the paper are verified against the notable laboratory and numerical experiments that capture the key aspects of canonical laminar and turbulent flows past a sphere. This analysis is supplemented with a study of challenging stably stratified flows past a sphere that verifies the NFTFV simulations against eminent experimental and numerical results. It demonstrates the potential of the advocated approach for research of intricate natural flows past complex terrain, combining dynamics of frictional and planetary boundary layers with gravity wave dynamics aloft.

Acknowledgements: This work was supported in part by the funding received from the European Research Council under the European Unions Seventh Framework Programme (FP7/2012/ERC Grant agreement no. 320375) and the Horizon 2020 Research and Innovation Programme (ESIWACE Grant agreement no. 675191 and ESCAPE Grant agreement no. 671627). 


\section{References}

[1] E. Achenbach, Vortex shedding from spheres, J. Fluid Mech. 62 (1974) 209221.

[2] D.P. Bacon, N.N. Ahmad, Z. Boybeyi, T.J. Dunn, M.S. Hall, P.C.S. Lee, R.A. Sarma, M.D. Turner, K.T. Waight, S.H. Young, J.W. Zack, A dynamically adapting weather and dispersion model: the operational multiscale environment model with grid adaptivity (OMEGA), Month. Weather Rev. 128 (2000) 20442067.

[3] P. Bagchi, S. Balachandar, Steady planar straining flow past a rigid sphere at moderate Reynolds number, J. Fluid Mech. 466 (2002) 365-407.

[4] G. Bouchet, M. Mebarek, J. Dušek, Hydrodynamic forces acting on a rigid fixed sphere in early transitional regimes, Eur. J. Mechanics-B/Fluids 25 (2006) 321336.

[5] G. Constantinescu, M. Chapelet, K. Squires, Turbulence modeling applied to flow over a sphere, AIAA Journal 41 (2003) 1733-1742.

[6] G. Constantinescu, K. Squires, LES and DES investigations of turbulent flow over a sphere at $\operatorname{Re}=10,000$, Flow, Turbulence and Combustion 70 (2003) 267298.

[7] G. Constantinescu, K. Squires, Numerical investigations of flow over a sphere in the subcritical and supercritical regimes, Phys. of Fluids 16 (2004) 1449-1466.

[8] R. Courant, D. Hilbert, Methods of Mathematical Physics, 2, Wiley-VCH, Mörlenbach, (1962).

[9] N.A. Crook, T.L. Clark, M.W. Moncrieff, The Denver Cyclone 1. generation in low Froude-number flow, J. Atmos. Sci. 47 (1990) 2725-2742.

[10] J.A. Domaradzki, Z. Xiao, P.K. Smolarkiewicz, Effective eddy viscosities in implicit large eddy simulations of turbulent flows, Phys. Fluids 15 (2003) 38903893.

[11] P.G. Drazin, On the steady flow of a fluid of variable density past obstacle, Tellus 13 (1961) 239-251.

[12] C.C. Epifanio, R. Rotunno, The dynamics of orographic wake formation in flows with upstream blocking, J. Atmos. Sci. 62 (2005) 3127-3150.

[13] B. Fornberg, A.R. Elcrat, Some observations regarding steady laminar flows past bluff bodies, Phil. Trans. R. Soc. A Math Phys Eng Sci. 372 (2014) Art No.20130353.

[14] B. Fornberg, Bengt, Steady viscous flow past a sphere at high Reynolds numbers, J. Fluid Mech. 190 (1988) 471-489.

[15] H. Hanazaki, A numerical study of 3-dimensional stratified flow past a sphere, J. Fluid Mech. 192 (1988) 393-419 
[16] R. Hassanzadeh, B. Sahin, M. Ozgoren, Numerical investigation of flow structures around a sphere, Int. J. Comput. Fluid Dynamics, 25 (2011) 535545 .

[17] Y. Hoarau, D. Pena, J.B. Vos, D. Charbonnier, A. Gehri, M. Braza, T. Deloze, E. Laurendau, Recent developments of the Navier Stokes Multi Block (NSMB) CFD solver, AIAA-Paper 2016-2056, (2016).

[18] J.C.R. Hunt, W.H. Snyder, Experiments on stably and neutrally stratified flow over a model three-dimensional hill, J. Fluid Mech. 96 (1980) 671-704.

[19] J. Jeong, F. Hussain, On the identification of a vortex, J. Fluid Mech. 285 (1995) 69-94.

[20] T.A. Johnson, V.C. Patel, Flow past a sphere up to a Reynolds number of 300, J. Fluid Mech. 378 (1999) 19-70.

[21] D.A. Jones, D.B. Clarke, Simulation of flow past a sphere using the Fluent code, Australian Government Department of Defence report, Defence Science and Technology Organization, (2008).

[22] Y. Kallinderis, H.T. Ahn, Incompressible Navier-Stokes method with general hybrid meshes, J. Comput. Phys. 210 (2005) 75-108.

[23] D. Kim, H. Choi, Large eddy simulation of turbulent flow over a sphere using an immersed boundary method, Third AFSOR international conference on direct numerical simulations and large eddy simulations (2001) DTIC Document, 7355-9, University of Texas Arlington-742.

[24] C. Kühnlein, P.K. Smolarkiewicz, An unstructured-mesh finite-volume MPDATA for compressible atmospheric dynamics, J. Comput. Phys. 334 (2017) $16-30$.

[25] B.P. Le Clair, A.E. Hamielec, H.R. Pruppacher, A numerical study of the drag on a sphere at low and intermediate Reynolds numbers, J. Atmos. Scien. 27 (1970) 308-315.

[26] Q. Lin, W.R. Lindberg, D.L. Boyer, H.J.S. Fernando, Stratified flow past a sphere, J. Fluid Mech. 240 (1992) 315-354.

[27] F.B. Lipps, R.S. Hemler, A scale analysis of deep moist convection and some related numerical calculations, J. Atmos. Sci. 39 (1982) 2192-2210.

[28] F.B. Lipps, On the anelastic approximation for deep convection, J. Atmos. Sci. 47 (1990) 1794-1798.

[29] K.E.B. Lofquist, L.P. Purtell, Drag on a sphere moving horizontally through a stratified liquid, J. Fluid Mech. 148 (1984) 271-284.

[30] J. Magnaudet, M. Rivero, J. Fabre, Accelerated flows past a rigid sphere or a spherical bubble. Part 1. Steady straining flow, J. Fluid Mech. 284 (1995) $97-135$. 
[31] L.G. Margolin, W.J. Rider, A rational for implicit turbulence modelling, International Journal for Numerical Methods in Fluids, 39 (2002) 821-841.

[32] M.D. Mikhailov, A.P. Silva Freire, The drag coefficient of a sphere: An approximation using Shanks transform, Powder Technology 237 (2013) 432-435.

[33] H.K. Moffat, The topological (as opposed to the analytical) approach to fluid and plasma flow problems, in Topological Fluid Mechanics, Proc. Symp. IUTAM, Cambridge, UK, H.K. Moffat and A. Tsinober, Eds., Cambridge University Press, 1-10.

[34] I. Nakamura, Steady wake behind a sphere, Phys. Fluids 19 (1976) 5-8.

[35] M. Ozgoren, E. Pinar, B. Sahin, H. Akilli, Comparison of flow structures in the downstream region of a cylinder and sphere, Int. J. Heat and Fluid Flow 32 (2011) 1138-1146.

[36] P. Ploumhans, G.S. Winckelmans, J.K. Salmon, A. Leonard, M.S. Warren, Vortex methods for direct numerical simulation of three-dimensional bluff body flows: application to the sphere at $\mathrm{Re}=300,500$, and 1000, J. Comput. Physics 178 (2002) 427-463.

[37] J.M. Prusa, P.K. Smolarkiewicz, A.A. Wyszogrodzki, EULAG, a computational model for multiscale flows, Comput. Fluids 37 (2008) 1193-1207.

[38] I. Rodriguez, O. Lehmkuhl, R. Borrell, A. Oliva, C.D. Pérez-Segarra, Direct numerical simulation of turbulent wakes: flow past a sphere at $R e=5000$, Fifth European Conference on Computational Fluid Dynamics Lisbon, Portugal 2010.

[39] H. Sakamoto, H. Haniu, A study on vortex shedding from spheres in a uniform flow, J. Fluids Eng. 112 (1990) 386-392.

[40] H. Sakamoto, H. Haniu, The formation mechanism and shedding frequency of vortices from a sphere in uniform shear flow, J. Fluid Mech. 287 (1995) 151-171.

[41] V. Seidl, S. Muzaferija, M. Perić, Parallel DNS with local grid refinement, Applied Scientific Research 59 (1997) 379-394.

[42] M. Shur, P.R. Spalart, M. Strelets, A. Travin, Detached-eddy simulation of an airfoil at high angle of attack, Engineering Turbulence Modelling and Measurement 4 Elsevier, (1999) 669-678.

[43] R.B. Smith, Linear theory of stratified hydrostatic flow past an isolated mountain, Tellus 32 (1980) 348-364.

[44] R.B. Smith, Linear theory of hydrostatic flow over an isolated mountain in isosteric coordinates, J. Atmos. Sci. 45 (1988) 3889-3896.

[45] P.A. Sheppard, Airflow over mountains, Q.J. Roy. Meteorol. Soc., 82 (1956) 528-529.

[46] P.K. Smolarkiewicz, R. Rasmussen, T.L. Clark, On the dynamics of Hawaiian rainbands: Island forcing, J. Atmos. Sci. 45 (1988) 1872-1905. 
[47] P.K. Smolarkiewicz, R. Rotunno, Low Froude number flow past threedimensional obstacles. Part I: Baroclinically generated lee vortices, J. Atmos. Sci. 46 (1989) 1154-1164.

[48] P.K. Smolarkiewicz, R. Rotunno, Low Froude number flow past threedimensional obstacles. Part II: Upwind flow reversal zone, J. Atmos. Sci. 47 (1990) 1498-1511.

[49] P.K. Smolarkiewicz, L.G. Margolin, On forward-in-time differencing for fluids: extension to a curvilinear framework. Month. Weather Rev. 121 (1993) 18471859.

[50] P.K. Smolarkiewicz, J. Szmelter, Multidimensional positive definite advection transport algorithm (MPDATA): an edge-based unstructured-data formulation, Int. J. Numer. Meth. Fluids 47 (2005) 1293-1299.

[51] P.K. Smolarkiewicz, J. Szmelter, MPDATA: An Edge-Based Unstructured-Grid Formulation, J. Comput. Phys. 206 (2005) 624-649.

[52] P.K. Smolarkiewicz, J. Szmelter, An MPDATA based solver for compressible flows, Int. J. Numer. Methods Fluids 56 (2008) 1529-1534.

[53] P.K. Smolarkiewicz, J. Szmelter, Iterated upwind schemes for gas dynamics, J. Comput. Phys. 228 (2009) 33-54.

[54] P.K. Smolarkiewicz, J. Szmelter, A nonhydrostatic unstructured-mesh soundproof model for simulation of internal gravity waves, Acta Geophysica 59 (2011) 1109-1134.

[55] P.K. Smolarkiewicz, P. Charbonneau, EULAG, a computational model for multiscale flows: An MHD extension, J. Comput. Phys. 236 (2013) 608-623.

[56] P.K. Smolarkiewicz, J. Szmelter, A.A. Wyszogrodzki, An unstructured-mesh atmospheric model for nonhydrostatic dynamics, J. Comput. Phys. 254 (2013) 184-199.

[57] P.K. Smolarkiewicz, W. Deconinck, M. Hamrud, C. Kühnlein, G. Mozdzynski, J. Szmelter, N.P. Wedi, A finite-volume module for simulating global all-scale atmospheric flows, J. Comput. Phys. 315 (2016) 287-304.

[58] P.K. Smolarkiewicz, J. Szmelter, F. Xiao, Simulation of all-scale atmospheric dynamics on unstructured meshes, J. Comput. Phys. 322 (2016) 267287.

[59] P.K. Smolarkiewicz, C. Kühnlein, W.W. Grabowski, A finite-volume module for cloud-resolving simulations of global atmospheric flows, J. Comput. Phys. 341 (2017) 208-229.

[60] P.R. Spalart, W.H. Jou, M. Strelets, S.R. Allmaras, Comments on the feasibility of LES for wings, and on a hybrid RANS/LES approach, in Liu C., Liu Z. (Eds.) Advances in LES/DNS, First AFOSR International Conference on DNS/LES. Greyden Press, Louisiana Tech University (1997). 
[61] P.R. Spalart, S.R. Allmaras, A one-equation turbulence model for aerodynamic flows, AIAA Paper 92-0439 (1992).

[62] S. Shirayama, Flow past a sphere-Topological transitions of the vorticity field, AIAA Journal 30 (1992) 349-358.

[63] J. Szmelter, P.K. Smolarkiewicz, MPDATA error estimator for mesh adaptivity, Int. J. Numer. Meth. Fluids 50 (2006) 1269-1293.

[64] J. Szmelter, P.K. Smolarkiewicz, An edge-based unstructured mesh discretisation in geospherical framework, J. Comput. Phys. 229 (2010) 49804995 .

[65] J. Szmelter, P.K. Smolarkiewicz, An edge-based unstructured mesh framework for atmospheric flows, Comput. Fluids. 46 (2011) 455-460.

[66] J. Szmelter, Z. Zhang, P.K. Smolarkiewicz, An unstructured-mesh atmospheric model for nonhydrostatic dynamics: Towards optimal mesh resolution, J. Comput. Phys. 294 (2015) 363-381.

[67] M. Tabata, K. Itakura, A precise computation of drag coefficients of a sphere, Int. J. of Comput. Fluid Dynamics 9 (1998) 303-311.

[68] S. Taneda, Experimental investigation of the wake behind a sphere at low Reynolds numbers, J. Phys. Soc. Japan, 11 (1956) 1104-1108.

[69] S. Taneda, Visual observations of the flow past a sphere at Reynolds numbers between $10^{4}$ and $10^{6}$, J. Fluid Mech. 85 (1978) 187-192.

[70] A.G. Tomboulides, S.A. Orszag, Numerical investigation of transitional and weak turbulent flow past a sphere, J. Fluid Mech. 416 (2000) 45-73.

[71] S.B. Vosper, Three-dimensional numerical simulations of strongly stratified flow past conical orography, J. Atmos. Sci. 57 (2000) 3716-3739. 